\title{
Grammar logicised: relativisation
}

\author{
Glyn Morrill ${ }^{1}$
}

Published online: 8 February 2017

(C) The Author(s) 2017. This article is published with open access at Springerlink.com

\begin{abstract}
Many variants of categorial grammar assume an underlying logic which is associative and linear. In relation to left extraction, the former property is challenged by island domains, which involve nonassociativity, and the latter property is challenged by parasitic gaps, which involve nonlinearity. We present a version of type logical grammar including 'structural inhibition' for nonassociativity and 'structural facilitation' for nonlinearity and we give an account of relativisation including islands and parasitic gaps and their interaction.
\end{abstract}

Keywords Islands · Parasitic gaps · Type logical categorial grammar · Relativisation · Structural facilitation $\cdot$ Structural inhibition

\section{Introduction}

Today mainstream linguistics is largely informal and computational linguistics is largely statistical. The task of spelling out completely explicit fragments seems either forgotten or taken too lightly. But there is a tradition of logical categorial grammar dating back to Ajdukiewicz (1935) which aims to practice linguistics as a branch of mathematical logic. For a while (especially around 1985-2000) this aspiration blended promisingly with the method of fragments, a methodology promoting the articulation of formal grammar fragments, such as the Montague fragment, and their combination and integration. But before major results were achieved in such comprehensive explicit integrated analysis, however, the field was overtaken by the aforementioned informal and statistical trends. Nevertheless, a small and committed community has remained.

Glyn Morrill

morrill@cs.upc.edu

1 Department of Computer Science, Universitat Politècnica de Catalunya, Barcelona 08034, Spain 
Now we are in a position to present a comprehensive explicit integrated analysis of relativisation as a show case example of deep and wide-coverage logical categorial grammar. ${ }^{1}$

'Categorial grammar' refers to a family of approaches to syntax and semantics in which grammatical information is lexicalised and expressions are classified by recursively defined syntactic types combinatorially governed by a type calculus, and in which semantic composition is driven by a structure preserving mapping from syntactic types to semantic types. In its type logical formulation the grammar is purely lexical and the type calculus is universal. There are a number of monographs, research monographs, reference works and textbooks on type logical grammar: Moortgat (1988, 1997), Morrill (1994, 2011b, 2012), Carpenter (1997), Jäger (2005), and Moot and Retoré (2012).

A major challenge to categorial grammar, and indeed to all approaches to grammar, is left extraction such as relativisation in which a fronted filler appears 'displaced' from a gap extraction site. Relativisation is an unbounded dependency phenomenon: the distance between a relative pronoun and its extraction site can be indefinitely long:

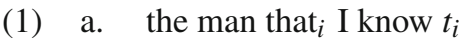

b. the man that ${ }_{i}$ you know I know $t_{i}$

c. the man that ${ }_{i}$ I know you know I know $t_{i}$

The treatment of relativisation in categorial grammar by means of assignment of higher-order functors to relative pronouns is well-established since Ades and Steedman (1982) and yields the unboundedness property through associative assembly of the body of a relative clause.

However, although relativisation is unbounded it is not unconstrained. Various 'islands' can inhibit or block relativisation: weak islands such as subjects and adverbial phrases, from which extraction is mildly unacceptable, and strong islands such as coordinate structures and relative clauses themselves, from which extraction is completely unacceptable (see e.g. Szabolcsi 2006):

(2) a. ?man who $i$ the friend of $t_{i}$ laughed

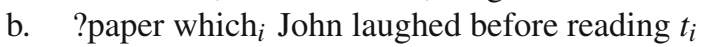

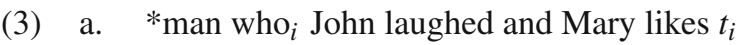

b. *man $\mathrm{who}_{i}$ John likes the woman that loves $t_{i}$

The conditions governing weak islands, especially, are subtle. For example, an indefinite appears to allow escape from a subject island:

(4) man $w_{i}$ a friend of $t_{i}$ laughed

\footnotetext{
1 In relation to the question whether this contribution is linguistic theory or language engineering, we think that the approach stands as the former without precluding the latter. For example, throughout we seek to avoid lexical ambiguity, which is problematic for language engineering, but nor, on our view, is appeal to lexical ambiguity a good thing from the point of view of linguistic theory, since it typically renegades on the capture of generalisations.
} 
And Kluender (1998) provides experimental evidence that there is a gradient effect on the acceptability of violations of the Complex Noun Phrase Constraint (CNPC) whereby increasing referential specificity of the extracted NP makes the sentence more acceptable, and so does decreasing the referential specificity of the extraction path. In general, under certain pragmatic or discourse-oriented conditions the processing of island violations is more acceptable (Lakoff 1986; Deane 1991; Kluender 1992, 1998; Kehler 2002; Hofmeister and Sag 2010).

This state of affairs raises the question of how to interface grammatical coverage with explanations in terms of information structure or semantic information, or processing. The facts are interpreted by Kubota and Levine (2015, Sect. 4.6.2) to argue for a version of logical categorial grammar that freely overgenerates island constraint 'violations' in the syntax. But, for example, Sprouse et al. (2012, p. 82) find "no evidence of a relationship between working memory capacity and island effects"; though see also the response of Hofmeister et al. (2013). On the other hand, Newmeyer (2016, p. 207) concedes that although the explanation of island phenomena has been a central feature of grammatical theory since its inception, "more and more syntacticians have concluded that an exclusively syntactic approach to islands is overly ambitious".

As we see it, the question of whether all, some or no island constraints should follow from syntactic theory is open, and while the jury is out on this issue it is acceptable, and we hope fruitful, to develop grammars as though islands were syntactic. Hence we shall adopt a conservative, syntactic, treatment of islands, while recognising that such 'structural inhibition' may need to be (re-)interpreted, for example, as a grammaticalisation of processing effects. ${ }^{2}$

Relativisation, furthermore, can also comprise 'parasitic extraction' in which a relative pronoun binds more than one extraction site (Taraldsen 1979; Engdahl 1983; Sag 1983). There is a single 'host' gap which is not in an island, and according to the received wisdom, and according with the terminology 'parasitic', this may license a 'parasitic' gap in (any number of immediate weak) islands:

(5) a. *the slave who ${ }_{i}$ John sold $t_{i} t_{i}$

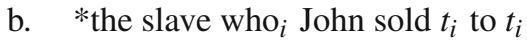

(6) a. the man who $i$ the friends of $t_{i}$ admire $t_{i}$

b. the paper which ${ }_{i}$ John filed $t_{i}$ without reading $t_{i}$

c. the paper which ${ }_{i}$ the editor of $t_{i}$ filed $t_{i}$ without reading $t_{i}$

In addition, we observe here that these parasitic gaps may in turn function as host gaps licensing further parasitic gaps in (weak) subislands, and so on recursively:

(7) a. man who $i$ the fact that the friends of $t_{i}$ admire $t_{i}$ surprises $t_{i}$

b. man who ${ }_{i}$ the fact that the friends of $t_{i}$ admire $t_{i}$ without praising $t_{i}$ offends $t_{i}$ without surprising $t_{i}$

There are examples in which there appears to be a parasitic gap which is not in an island. The following is example (8a) from Postal (1993):

\footnotetext{
2 See also Morrill (2000) for a way to link the binarity of formal categorial grammars and the gradience of the object of study.
} 


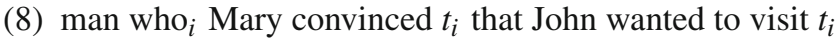

And an anonymous referee points out:

(9) people whom $i$ you sent pictures of $t_{i}$ to $t_{i}$

In respect of such examples we suggest that although there seems to be no island, there could be one. ${ }^{3}$ We present a tentative account along this line of 'optional islands' in Sect. 6.

Parasitic 'structural facilitation' represents a challenge to categorial grammar and all approaches to grammar. The above is the empirical analysis of islands and parasitic gaps and their interaction given a type logical, i.e. purely lexical, categorial account in Morrill (2011b, Chap. 5). In this paper we give an account of the empirical analysis which improves on that account in the following respects:

- Multimodality and associated multimodal structural postulates are removed. ${ }^{4}$

- The proposal is set in the context of the displacement calculus of Morrill and Valentín (2010) and Morrill et al. (2011) which is an advance on the discontinuous Lambek calculus of Morrill (2011b, Chap. 6).

- Nonlinearity (structural facilitation) is formalised by use of a 'stoup' (Girard 2011) which reduces the size of the proof search space.

- The rule of contraction generating parasitic gaps is simplified.

- The account integrates other aspects of grammatical analysis such as polymorphism, features, and intensionality.

- The correct interaction of all the grammatical aspects is verified by computergeneration of the analyses.

- Various possible exceptions to the empirical analysis are addressed.

The result, we think, is a formal and mathematically principled empirically adequate formalisation of relativisation which is thorough, very high level (concise) and which is computer-verified.

The structure of the paper is as follows. In Sect. 2 we present and illustrate the sequent calculus for our displacement logic. In Sect. 3 we present initial examples of analysis. In Sect. 4 we discuss approaches to relativisation with which we differ, and in Sect. 5 we present our theoretical analysis of relativisation. In Sect. 6 we address possible exceptions to our account. We conclude in Sect. 7. The semantic representation language used here is defined in Appendix 1, and a lexicon is given in Appendix 2.

\section{Framework}

The formalism used comprises the connectives of Table 1 . The heart of the logic is the displacement calculus of Morrill and Valentín (2010) and Morrill et al. (2011) made

\footnotetext{
3 Tom Roeper, p.c.

4 Structural postulates increase both the derivation search space and derivation length. Of course ways may be found to ameliorate this, but that would be precisely to absorb the structural properties in the way that is already done here (Valentín 2014). Ceteris paribus, given the choice between structural postulates or no structural postulates, the latter is to be preferred.
} 
Table 1 Categorial connectives

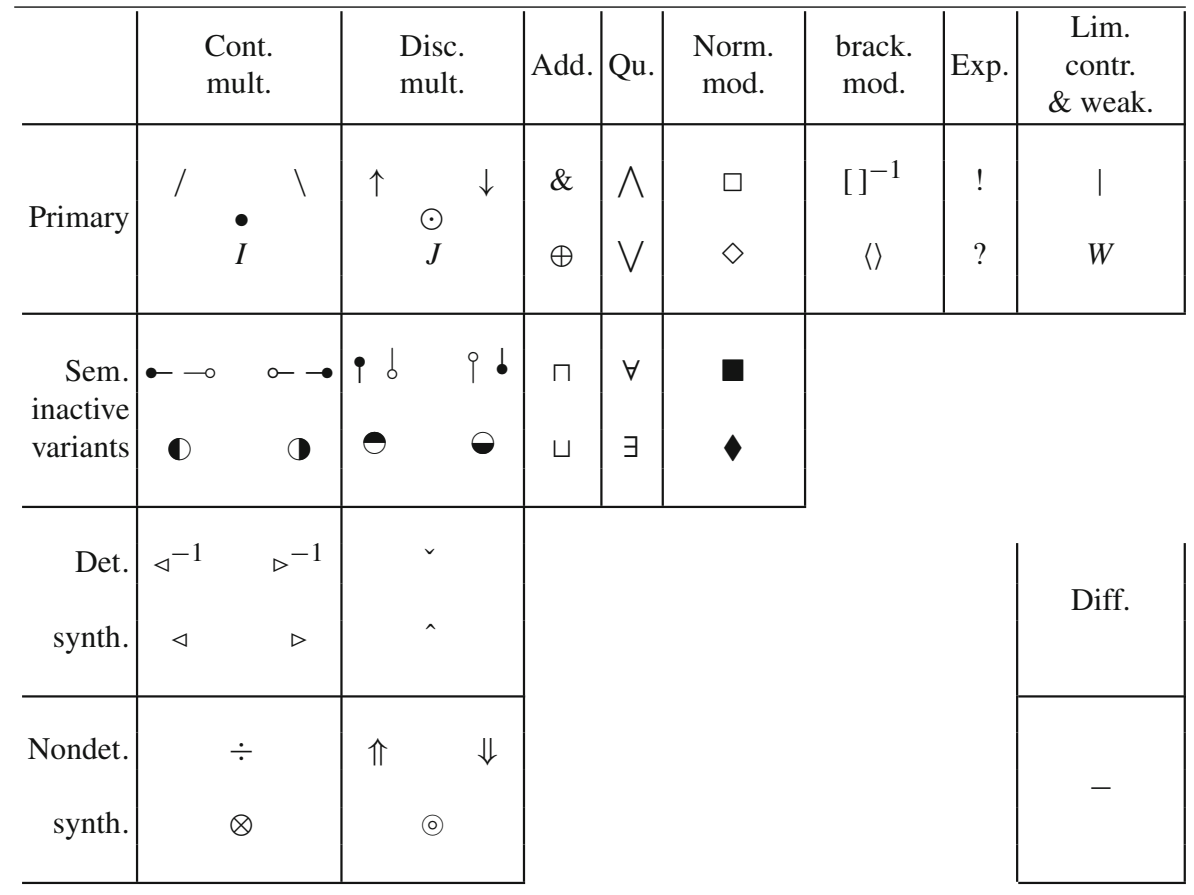

up of twin continuous and discontinuous residuated families of connectives having a pure Gentzen sequent calculus-without labels and free of structural rules-and enjoying Cut-elimination (Valentín 2012). Other primitive connectives are additives, 1 st order quantifiers, normal (i.e. distributive) modalities, bracket (i.e. nondistributive) modalities, exponentials, limited contraction and limited weakening, and difference. ${ }^{5}$

We can draw a clear distinction between the primitive connectives, and the semantically inactive connectives and the synthetic connectives which are abbreviatory and are there for convenience, and to simplify derivation. There are semantically inactive variants of the continuous and discontinuous multiplicatives, and semantically inactive variants of the additives, 1 st order quantifiers, and normal modalities. ${ }^{6}$ Synthetic connectives (Girard 2011) divide into the continuous and discontinuous deterministic

\footnotetext{
5 Once Cut-elimination is established, the only challenge to decidability comes from nonlinearity: the universal and existential exponentials. In this connection, Morrill and Valentín (2015a) introduced a displacement logic Db!? with a relevant modality ! without bracket conditioning, and another system $\mathbf{D b} \mathbf{b}_{\mathbf{b}}$ ? with bracket conditioning, as here. Kanovich et al. (2016) prove the undecidability of Db!? and in unpublished work announce the undecidability of $\mathbf{D b} !_{\mathbf{b}}$ ?. But Morrill and Valentín (2015a) prove the decidability of a linguistically sufficient special case of constrained bracketing of contraction with bracket conditioning.

6 For example, the semantically inactive additive conjunction $A \sqcap B: \phi$ abbreviates $A \& B:(\phi, \phi)$.
} 
Table 2 Syntactic types

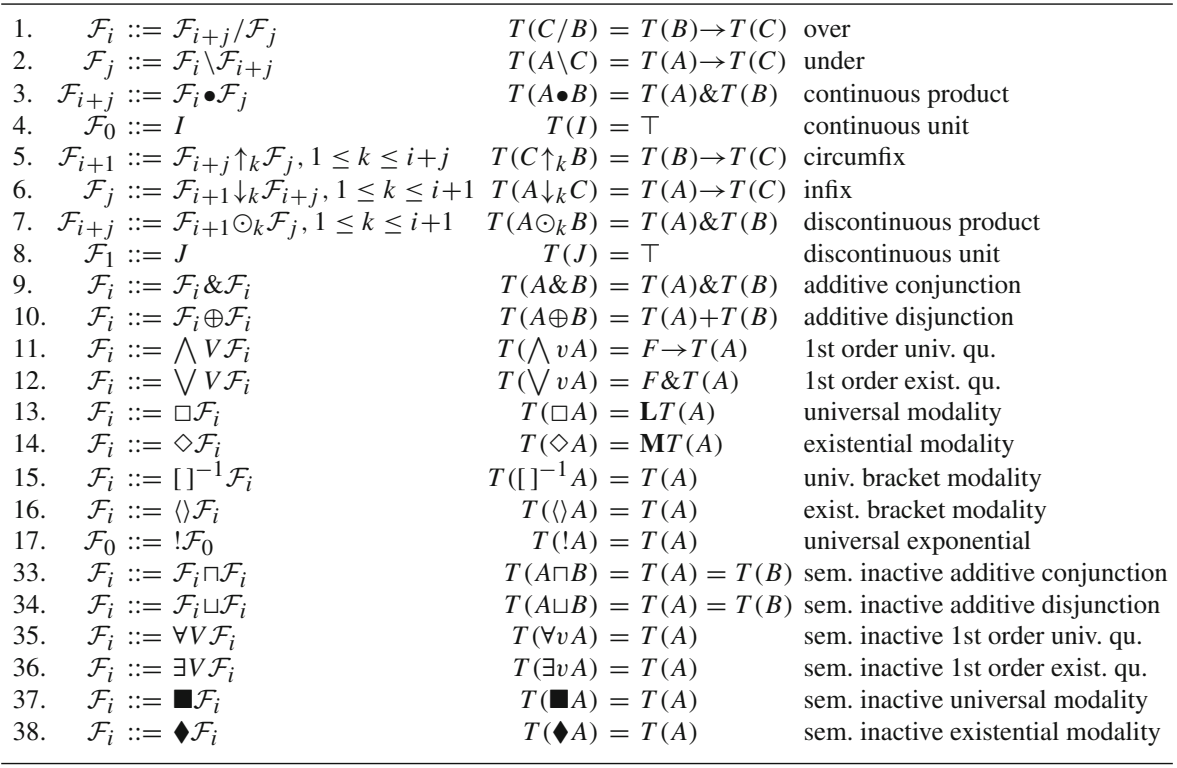

(unary) synthetic connectives, and the continuous and discontinuous nondeterministic (binary) synthetic connectives. ${ }^{7}$

\subsection{Syntactic types}

The syntactic types of displacement logic are sorted $\mathcal{F}_{0}, \mathcal{F}_{1}, \mathcal{F}_{2}, \ldots$ according to the number of points of discontinuity $0,1,2, \ldots$ their expressions contain. Each type predicate letter has a sort and an arity which are naturals, and a corresponding semantic type. Assuming ordinary terms to be already given, where $P$ is a type predicate letter of sort $i$ and arity $n$ and $t_{1}, \ldots, t_{n}$ are terms, $P t_{1} \ldots t_{n}$ is an (atomic) type of sort $i$ of the corresponding semantic type. Compound types are formed by connectives as indicated in Table $2,{ }^{8}$ and the structure preserving semantic type map $T$ associates these with semantic types, where $F$ is a domain of feature values.

\subsection{Gentzen sequent calculus}

We use a Gentzen sequent presentation standard from Gentzen (1934) and Lambek (1958). (Labelled) natural deduction can proportion a congenial proof format for categorial logic because the compositional term-structure of Curry-Howard semantics

\footnotetext{
7 For example, the nondeterministic continuous division $B \div A$ abbreviates $(A \backslash B) \sqcap(B / A)$.

8 We list only connectives drawn from the first two rows of Table 1, and we omit some which are not central here.
} 
follows the structure of natural deduction derivation. However, here we use the Gentzen sequent proof format because:

- Natural deduction does not capture symmetries as satisfactorily as Gentzen sequent calculus. For example, while product right is easy to express in ND, product left is awkward (unnatural); but both are straightforwardly expressed in Gentzen sequent calculus. ${ }^{9}$

- The title of the paper is 'Grammar logicised', i.e. there is an emphasis on the thesis that grammar can be reduced to logic. To maintain this it is appropriate to pitch the logical aspects as closely as possible to the usual Gentzen format with the associated symmetries and metatheory.

- It enables uniform formulation of all of the rules of inference.

- It dispenses with phonological labels.

- It lends itself more transparently to standard proof of Cut-elimination and consequent decidability results.

- It lends itself more transparently to focalisation (Andreoli 1992; Morrill and Valentín 2015b) and consequent efficient computer generation and verification of the analyses.

Although discontinuous types play a minor role here, our analysis is pitched in their context in order to show its consistency with displacement calculus. Crucially, in Gentzen sequent configurations $(\Gamma, \Delta)$ for displacement calculus a discontinuous type is a mother, rather than a leaf, and dominates its discontinuous components marked off by curly brackets and colons.

In Gentzen sequent antecedents for displacement logic with bracket modalities (structural inhibition) and exponentials (structural facilitation) there is also a bracket constructor for the former and 'stoups' for the latter.

Stoups (cf. the linear logic of Girard 2011) $(\zeta)$ are stores read as sets for re-usable (nonlinear) resources which appear at the left of a configuration marked off by a semicolon (when the stoup is empty the semicolon may be ommited). The stoup of linear logic is for resources which can be contracted (copied) or weakened (deleted). By contrast, our stoup is for a linguistically motivated variant of contraction, and does not allow weakening. Furthermore, whereas linear logic is commutative, our logic is in general noncommutative and the stoup is used for resources which are also commutative. To anticipate our analysis a little, a hypothetical subtype emitted by a relative pronoun corresponding to a long-distance dependency will enter a stoup, percolate in stoups, maybe contracting to create (parasitic) gaps, and finally permute into a (host) extraction site.

9 Thus, in Gentzen format product left is simply $\frac{\Delta(A, B) \Rightarrow D}{\Delta(A \bullet B) \Rightarrow D}$ but unlabelled ND requires something like

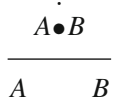

which does not respect the single-conclusion condition and there are consequent complications regarding, for example, which hypotheses can be cancelled when. 
A configuration together with a stoup is a zone $(\Xi)$. The bracket constructor applies not to a configuration alone but to a configuration with a stoup, i.e a zone: reusable resources are specific to their domain.

Stoups $\mathcal{S}$ and configurations $\mathcal{O}$ are defined by the following ( $\varnothing$ is the empty stoup; $\Lambda$ is the empty configuration; the separator 1 marks points of discontinuity: ${ }^{10}$

$$
\begin{aligned}
\text { (10) } & \mathcal{S}::=\emptyset \mid \mathcal{F}_{0}, \mathcal{S} \\
\mathcal{O} & ::=\Lambda \mid \mathcal{T}, \mathcal{O} \\
\mathcal{T} & ::=1\left|\mathcal{F}_{0}\right| \mathcal{F}_{i>0}\{\underbrace{\mathcal{O}: \ldots: \mathcal{O}}_{i \mathcal{O}^{\prime} \mathrm{s}}\} \mid[\mathcal{S} ; \mathcal{O}]
\end{aligned}
$$

For a type $A$, its sort $s(A)$ is the $i$ such that $A \in \mathcal{F}_{i}$. For a configuration $\Gamma$, its sort $s(\Gamma)$ is $|\Gamma|_{1}$, i.e. the number of points of discontinuity 1 which it contains. Sequents are of the form:

(11) $\mathcal{S} ; \mathcal{O} \Rightarrow \mathcal{F}$ such that $s(\mathcal{O})=s(\mathcal{F})$

The figure $\vec{A}$ of a type $A$ is defined by:

(12) $\vec{A}= \begin{cases}A & \text { if } s(A)=0 \\ A\{\underbrace{1: \ldots: 1}_{s(A) 1^{\prime} \mathrm{s}}\} & \text { if } s(A)>0\end{cases}$

Where $\Gamma$ is a configuration of sort $i$ and $\Delta_{1}, \ldots, \Delta_{i}$ are configurations, the fold $\Gamma \otimes\left\langle\Delta_{1}: \ldots: \Delta_{i}\right\rangle$ is the result of replacing the successive 1's in $\Gamma$ by $\Delta_{1}, \ldots, \Delta_{i}$ respectively. Where $\Gamma$ is of sort $i$, the hyperoccurrence notation $\Delta\langle\Gamma\rangle$ abbreviates $\Delta_{0}\left(\Gamma \otimes\left\langle\Delta_{1}: \ldots: \Delta_{i}\right\rangle\right)$, i.e. a context configuration $\Delta$ (which is externally $\Delta_{0}$ and internally $\Delta_{1}, \ldots, \Delta_{i}$ ) with a potentially discontinuous distinguished subconfiguration $\Gamma$ (continuous if $i=0$, discontinuous if $i>0$ ).

Where $\Delta$ is a configuration of sort $i>0$ and $\Gamma$ is a configuration, the $k$ th metalinguistic intercalation $\left.\Delta\right|_{k} \Gamma, 1 \leq k \leq i$, is given by:

$$
\left.\Delta\right|_{k} \Gamma=d_{f} \Delta \otimes\langle\underbrace{1: \ldots: 1}_{k-1 \text { 1's }}: \Gamma: \underbrace{1: \ldots: 1}_{i-k 1 \text { 's }}\rangle
$$

i.e. $\left.\Delta\right|_{k} \Gamma$ is the configuration resulting from replacing by $\Gamma$ the $k$ th separator in $\Delta$.

\subsection{Rules and linguistic applications}

A semantically labelled sequent is a sequent in which the antecedent type occurrences $A_{1}, \ldots, A_{n}$ are labelled by distinct variables $x_{1}, \ldots, x_{n}$ of types $T\left(A_{1}\right), \ldots, T\left(A_{n}\right)$ respectively, and the succedent type $A$ is labelled by a term of type $T(A)$ with free variables drawn from $x_{1}, \ldots, x_{n}$. In this section we give the semantically labelled Gentzen sequent rules for some primitive connectives, and indicate some linguistic applications.

The continuous multiplicatives of Fig. 1, the Lambek connectives, Lambek (1958, 1988), defined in relation to concatenation/appending, are the basic means of categorial

\footnotetext{
10 Note that only types of sort 0 can go into the stoup; reusable types of other sorts would not preserve the sequent antecedent-succedent sort equality under contraction: $0+0=0$, but $i+i \neq i$ for $i>0$.
} 
1. $\frac{\left.\zeta_{1} ; \Gamma \Rightarrow B: \psi \quad \zeta_{2} ; \Delta \overrightarrow{\langle C}: z\right\rangle \Rightarrow D: \omega}{\zeta_{1} \uplus \zeta_{2} ; \Delta\langle\overrightarrow{C / B}: x, \Gamma\rangle \Rightarrow D: \omega\{(x \psi) / z\}} / L \quad \frac{\zeta ; \Gamma, \vec{B}: y \Rightarrow C: \chi}{\zeta ; \Gamma \Rightarrow C / B: \lambda y \chi} / R$

2. $\quad \frac{\zeta_{1} ; \Gamma \Rightarrow A: \phi \quad \zeta_{2} ; \Delta\langle\vec{C}: z\rangle \Rightarrow D: \omega}{\zeta_{1} \uplus \zeta_{2} ; \Delta\langle\Gamma, \overrightarrow{A \backslash C}: y\rangle \Rightarrow D: \omega\{(y \phi) / z\}} \backslash L \quad \frac{\zeta ; \vec{A}: x, \Gamma \Rightarrow C: \chi}{\zeta ; \Gamma \Rightarrow A \backslash C: \lambda x \chi} \backslash R$

3. $\frac{\Xi\langle\vec{A}: x, \vec{B}: y\rangle \Rightarrow D: \omega}{\Xi\langle\overrightarrow{A \bullet B}: z\rangle \Rightarrow D: \omega\left\{\pi_{1} z / x, \pi_{2} z / y\right\}} \bullet L \quad \frac{\zeta_{1} ; \Gamma_{1} \Rightarrow A: \phi \quad \zeta_{2} ; \Gamma_{2} \Rightarrow B: \psi}{\zeta_{1} \uplus \zeta_{2} ; \Gamma_{1}, \Gamma_{2} \Rightarrow A \bullet B:(\phi, \psi)} \bullet R$

4. $\frac{\Xi\langle\Lambda\rangle \Rightarrow A: \phi}{\Xi\langle\vec{I}: x\rangle \Rightarrow A: \phi} I L \quad \frac{\emptyset ; \Lambda \Rightarrow I: 0}{I R}$

Fig. 1 Continuous multiplicatives

5. $\quad \frac{\zeta_{1} ; \Gamma \Rightarrow B: \psi \quad \zeta_{2} ; \Delta\langle\vec{C}: z\rangle \Rightarrow D: \omega}{\zeta_{1} \uplus \zeta_{2} ; \Delta\left\langle\overrightarrow{C \uparrow_{k} B}:\left.x\right|_{k} \Gamma\right\rangle \Rightarrow D: \omega\{(x \psi) / z\}} \uparrow_{k} L \quad \frac{\zeta ;\left.\Gamma\right|_{k} \vec{B}: y \Rightarrow C: \chi}{\zeta ; \Gamma \Rightarrow C \uparrow_{k} B: \lambda y \chi} \uparrow_{k} R$

6. $\quad \frac{\zeta_{1} ; \Gamma \Rightarrow A: \phi \quad \zeta_{2} ; \Delta\langle\vec{C}: z\rangle \Rightarrow D: \omega}{\zeta_{1} \uplus \zeta_{2} ; \Delta\left\langle\left.\Gamma\right|_{k} \overrightarrow{A \downarrow_{k} C}: y\right\rangle \Rightarrow D: \omega\{(y \phi) / z\}} \downarrow_{k} L \quad \frac{\zeta ; \vec{A}:\left.x\right|_{k} \Gamma \Rightarrow C: \chi}{\zeta ; \Gamma \Rightarrow A \downarrow_{k} C: \lambda x \chi} \downarrow_{k} R$

7. $\frac{\Xi\left\langle\vec{A}:\left.x\right|_{k} \vec{B}: y\right\rangle \Rightarrow D: \omega}{\Xi\left\langle\overrightarrow{A \odot_{k} B}: z\right\rangle \Rightarrow D: \omega\left\{\pi_{1} z / x, \pi_{2} z / y\right\}} \odot_{k} L \quad \frac{\zeta_{1} ; \Gamma_{1} \Rightarrow A: \phi \quad \zeta_{2} ; \Gamma_{2} \Rightarrow B: \psi}{\zeta_{1} \uplus \zeta_{2} ;\left.\Gamma_{1}\right|_{k} \Gamma_{2} \Rightarrow A \odot_{k} B:(\phi, \psi)} \odot_{k} R$

8. $\frac{\Xi\langle 1\rangle \Rightarrow A: \phi}{\Xi\langle\vec{J}: x\rangle \Rightarrow A: \phi} J L \quad \overline{\emptyset ; 1 \Rightarrow J: 0} J R$

Fig. 2 Discontinuous multiplicatives

categorization and subcategorization. Note that here and throughout the active types in antecedents are figures (vectorial) whereas those in succedents are not; intuitively this is because antecedents are structured but succedents are not. The directional divisions over, /, and under, \, are exemplified by assignments such as the: $N / C N$ for the man: $N$ and sings: $N \backslash S$ for John sings: $S$, and loves: $(N \backslash S) / N$ for John loves Mary: $S$. The continuous product $\bullet$ is exemplified by a 'small clause' assignment such as considers: $(N \backslash S) /(N \bullet(C N / C N))$ for say John considers Mary socialist: $S .{ }^{11}$

The discontinuous multiplicatives of Fig. 2, the displacement connectives, Morrill and Valentín (2010) and Morrill et al. (2011), are defined in relation to intercalation/plugging. When the value of the $k$ subindex indicates the first (leftmost) point of discontinuity it may be omitted. Circumfixation, $\uparrow$, is exemplified by

\footnotetext{
${ }^{11}$ But this makes no different empirical predictions from the more standard type of analysis in categorial grammar which simply treats verbs like consider as taking a noun phrase and an infinitive. Products are more truly motivated by antecedent occurrences in the (continuous) analysis of past participles of Morrill (2011b, pp. 64-65), or the discontinuous generalisation of this for a past participle such as loved in Appendix 2 here.
} 
9. $\frac{\Xi\langle\vec{A}: x\rangle \Rightarrow C: \chi}{\Xi\langle\overrightarrow{A \& B}: z\rangle \Rightarrow C: \chi\left\{\pi_{1} z / x\right\}} \& L_{1} \quad \frac{\Xi\langle\vec{B}: y\rangle \Rightarrow C: \chi}{\Xi\langle\overrightarrow{A \& B}: z\rangle \Rightarrow C: \chi\left\{\pi_{2} z / y\right\}} \& L_{2}$

$$
\frac{\Xi \Rightarrow A: \phi \quad \Xi \Rightarrow B: \psi}{\Xi \Rightarrow A \& B:(\phi, \psi)} \& R
$$

10. $\quad \frac{\Xi\langle\vec{A}: x\rangle \Rightarrow C: \chi_{1} \quad \Xi\langle\vec{B}: y\rangle \Rightarrow C: \chi_{2}}{\Xi\langle\overrightarrow{A \oplus B}: z\rangle \Rightarrow C: z \rightarrow x \cdot \chi_{1} ; y \cdot \chi_{2}} \oplus L$

$$
\frac{\Xi \Rightarrow A: \phi}{\Xi \Rightarrow A \oplus B: \iota_{1} \phi} \oplus R_{1} \quad \frac{\Xi \Rightarrow B: \psi}{\Xi \Rightarrow A \oplus B: \iota_{2} \psi} \oplus R_{2}
$$

Fig. 3 Additives

a discontinuous idiom assignment gives $+1+$ the + cold + shoulder: $(N \backslash S) \uparrow N$ for Mary gives John the cold shoulder: $S$, and infixation, $\downarrow$, and circumfixation together are exemplified by a quantifier phrase assignment everyone: $(S \uparrow N) \downarrow S$ simulating Montague's S14 treatment of quantifying in. Circumfixation and discontinuous product, $\odot$, are illustrated together with the continuous unit in an assignment to a relative pronoun that: $(C N \backslash C N) /((S \uparrow N) \odot I)$ allowing both peripheral and medial extraction: that John likes: $C N \backslash C N$ and that John saw today: $C N \backslash C N$, although we will argue in Sect. 4 that this strategy is inadequate, and the main point of the present paper is to promote another approach to relativisation.

In relation to the multiplicative rules, notice how the stoup is distributed reading bottom-up from conclusions to premise: it is partitioned between the two premises in the case of binary rules, copied to the premise in the case of unary rules, and empty in the case of nullary rules (axioms).

The additives of Fig. 3, Lambek (1961), Morrill (1990a), Kanazawa (1992), have application to polymorphism. For example the additive conjunction \& can be used for rice: $N \& C N$ as in rice grows: $S$ and the rice grows: $S,{ }^{12}$ and the additive disjunction $\oplus$ can be used for is: $(N \backslash S) /(N \oplus(C N / C N))$ as in Tully is Cicero: $S$ and Tully is humanist: $S$. The additive disjunction can be used together with the continuous unit to express the optionality of a complement as in eats: $(N \backslash S) /(N \oplus I)$ for John eats fish: $S$ and John eats: $S .^{13}$

Notice how the stoup is identical in conclusions and premises of additive rules.

The quantifiers of Fig. 4, Morrill (1994), have application to features. For example, singular and plural number in sheep: $\bigwedge n C N n$ for the sheep grazes: $S$ and the sheep graze: $S$. And for a past, present or future tense finite sentence complement we can

\footnotetext{
12 Note the computational advantage of this approach over assuming an empty determiner: if empty operators were allowed they could potentially occur any number of times in any positions.

13 Note the advantage of this over simply listing intransitive and transitive lexical entries: empirically the latter does not capture the generalisation that in both cases the verb eats combines with a subject to the left, and computationally every lexical ambiguity doubles the lexical insertion search space. Appeal to lexical ambiguity constitutes resignation from the capture of generalisations and is at best a promissory solution, unless there is true ambiguity.
} 


$$
\begin{array}{lll}
\text { 11. } & \frac{\Xi\langle\overrightarrow{A[t / v]}: x\rangle \Rightarrow B: \psi}{\Xi\langle\widehat{\bigwedge v A}: z\rangle \Rightarrow B: \psi\{(z t) / x\}} \wedge L & \frac{\Xi \Rightarrow A[a / v]: \phi}{\Xi \Rightarrow \bigwedge v A: \lambda v \phi} \wedge R^{\dagger} \\
\text { 12. } & \frac{\Xi\langle\overrightarrow{A[a / v]}: x\rangle \Rightarrow B: \psi}{\Xi\langle\overrightarrow{\bigvee v A}: z\rangle \Rightarrow B: \psi\left\{\pi_{2} z / x\right\}} \vee L^{\dagger} & \frac{\Xi \Rightarrow A[t / v]: \phi}{\Xi \Rightarrow \bigvee v A:(t, \phi)} \vee R
\end{array}
$$

Fig. 4 Quantifiers, where ${ }^{\dagger}$ indicates that there is no $a$ in the conclusion

Fig. 5 Normal modalities, where $\mathbb{Q} / \diamond$ marks a structure all the types of which have main connective a box/diamond

Fig. 6 Bracket modalities
13.

$$
\frac{\Xi\langle\vec{A}: x\rangle \Rightarrow B: \psi}{\Xi\langle\overrightarrow{\square A}: z\rangle \Rightarrow B: \psi\left\{\left\{^{\vee} z / x\right\}\right.} \square L \quad \frac{\otimes \Xi \Rightarrow A: \phi}{\otimes \Xi \Rightarrow \square A:{ }^{\wedge} \phi} \square R
$$

14.

$$
\frac{\otimes \Xi\langle\vec{A}: x\rangle \Rightarrow \oplus B: \psi}{\otimes \Xi\langle\overrightarrow{\diamond A}: z\rangle \Rightarrow \oplus B: \psi\left\{\cup^{\cup} z / x\right\}} \diamond L \frac{\Xi \Rightarrow A: \phi}{\Xi \Rightarrow \diamond A:{ }^{\cap} \phi} \diamond R
$$

15.

$$
\begin{gathered}
\frac{\Xi\langle\vec{A}: x\rangle \Rightarrow B: \psi}{\Xi\left\langle\left[\overrightarrow{[]^{-1} A}: x\right]\right\rangle \Rightarrow B: \psi}[]^{-1} L \frac{[\Xi] \Rightarrow A: \phi}{\Xi \Rightarrow[]^{-1} A: \phi}[]^{-1} R \\
\Xi\langle[\vec{A}: x]\rangle \Rightarrow B: \psi \\
\frac{\Xi\langle\overrightarrow{\langle\rangle}: x\rangle \Rightarrow B: \psi}{\Xi \Rightarrow A: \phi} \quad \frac{}{[\Xi] \Rightarrow\langle\rangle A: \phi}\langle\rangle R
\end{gathered}
$$

have said: $(N \backslash S) / \bigvee t S f(t)$ in John said Mary walked: $S$, John said Mary walks: $S$ and John said Mary will walk: $S$.

Notice how the stoup is identical in conclusion and premise in each quantifier rule.

With respect to the normal modalities of Fig. 5, the universal (Morrill 1990b) has application to intensionality. For example, for a propositional attitude verb such as believes we can assign type $\square((N \backslash S) / \square S)$ with a modality outermost since the word has a sense, and a modality on the first argument but not the second, since the sentential complement is an intensional domain, but the subject is not.

Notice how the stoup is identical in conclusion and premise in each normal modality rule.

The bracket modalities of Fig. 6, Morrill (1992) and Moortgat (1995), have application to nonassociativity and syntactical domains such as prosodic phrases and extraction islands. For example, single bracketing for weak islands: walks: \langle\rangle $N \backslash S$ for the subject condition, and without: [ $]^{-1}(V P \backslash V P) / V P$ for the adverbial island constraint; and double bracketing for strong islands such as and: $\left(S \backslash[]^{-1}[]^{-1} S\right) / S$ for the coordinate structure constraint.

Notice how the stoup is identical in conclusions and premises of bracket modality rules.

Finally, there is nonlinearity. The universal exponential of Fig. 7, Girard (1987), Barry et al. (1991), Morrill (1994), and Morrill and Valentín (2015a) has application to parasitic extraction. In the formulation here $! L$ moves the operand of a universal 


$$
\text { 17. } \begin{aligned}
& \frac{\Xi\left(\zeta \uplus\{A: x\} ; \Gamma_{1}, \Gamma_{2}\right) \Rightarrow B: \psi}{\Xi\left(\zeta ; \Gamma_{1}, ! A: x, \Gamma_{2}\right) \Rightarrow B: \psi} ! L \quad \frac{\zeta ; \Lambda \Rightarrow A: \phi}{\zeta ; \Lambda \Rightarrow ! A: \phi} ! R \\
& \frac{\Xi\left(\zeta ; \Gamma_{1}, A: x, \Gamma_{2}\right) \Rightarrow B: \psi}{\Xi\left(\zeta \uplus\{A: x\} ; \Gamma_{1}, \Gamma_{2}\right) \Rightarrow B: \psi} ! P \\
& \frac{\Xi\left(\zeta \uplus\{A: x\} ; \Gamma_{1},\left[\{A: y\} ; \Gamma_{2}\right], \Gamma_{3}\right) \Rightarrow B: \psi}{\Xi\left(\zeta \uplus\{A: x\} ; \Gamma_{1}, \Gamma_{2}, \Gamma_{3}\right) \Rightarrow B: \psi\{x / y\}} ! C
\end{aligned}
$$

Fig. 7 Universal exponential

exponential (e.g. the hypothetical subtype of relativisation) into the stoup, where it will percolate as commented for the above rules. From there it can be copied into the stoup of a newly-created bracketed domain by the contraction rule ! $C$ (producing a parasitic gap), and it can be moved into any position in the matrix configuration of its zone by $! P$ (producing a normal nonparasitic or host gap). For example:

$$
\begin{gathered}
\frac{\ldots, A, \ldots,[\ldots, A, \ldots], \ldots, \ldots \Rightarrow D}{\ldots, A, \ldots,[A ; \ldots, \ldots], \ldots, \ldots \Rightarrow D} ! P \\
\frac{A ; \ldots, \ldots,[A ; \ldots, \ldots], \ldots, \ldots \Rightarrow D}{\vdots} ! P \\
\frac{A ; \ldots, \ldots, \ldots, \ldots, \ldots, \ldots \Rightarrow B}{\ldots, \ldots, \ldots, \ldots, \ldots, A, \ldots \Rightarrow B} ! L
\end{gathered}
$$

Reading upwards, first the $! A$ is moved into the stoup by $! L$ and the exponential modality is removed (being in the stoup means that the type is under the associated resource management regime). We assume some derivation steps, indicated by vertical dots, and then an application of contraction !C. A domain becomes bracketed, and this domain contains A in its stoup. This would correspond to a weak island containing a parasitic gap. Finally the 'host' and 'parasitic' gaps are permuted into position by two applications of $! P$.

Using the universal exponential, !, for which contraction induces island brackets, we can assign a relative pronoun type that: $(C N \backslash C N) /(S / ! N)$ allowing parasitic extraction such as paper that John filed without reading: $C N$, where parasitic gaps can appear only in (weak) islands, but can be iterated in subislands, for example, man who the fact that the friends of admire without praising surprises. See Sect. 5.

Crucially, in the linguistic formulation ! does not have weakening, i.e. deletion, since, e.g., the body of a relative clause must contain a gap: *man who John loves Mary.

In relation to the rest of the primary connectives: the existential exponential ? has application to iterated coordination (Morrill 1994; Morrill and Valentín 2015a) and (unboundedly iterated) respectively (Morrill and Valentín 2016), the limited contraction | of Jäger (2005) has application to anaphora and the limited weakening $W$ of Morrill and Valentín (2014b) has application to words as types. The remaining, semantically inactive, connectives listed here were introduced as follows. Semanti- 
Fig. 8 Semantically inactive additive conjunction
Fig. 9 Semantically inactive universal quantifier, where $\dagger$ indicates that there is no $a$ in the conclusion

$$
\begin{aligned}
& \text { 35. } \frac{\Xi\langle\overrightarrow{A[t / v]}: x\rangle \Rightarrow B: \psi}{\Xi\langle\overrightarrow{\forall v A}: x\rangle \Rightarrow B: \psi} \forall L \frac{\Xi \Rightarrow A[a / v]: \phi}{\Xi \Rightarrow \forall v A: \phi} \forall R^{\dagger} \\
& \text { 37. } \frac{\Xi\langle\vec{A}: x\rangle \Rightarrow B: \psi}{\Xi\langle\vec{\square}: x\rangle \Rightarrow B: \psi} \square \frac{\square / \square \Xi \Rightarrow A: \phi}{\square / \square \Xi \Rightarrow \square A: \phi} \square R
\end{aligned}
$$

Fig. 10 Semantically inactive universal normal modality

$$
\text { 33. } \begin{aligned}
& \frac{\Xi\langle\vec{A}: x\rangle \Rightarrow C: \chi}{\Xi\langle\overrightarrow{A \sqcap B}: x\rangle} \Rightarrow C: \chi \sqcap L_{1} \quad \frac{\Xi\langle\vec{B}: y\rangle \Rightarrow C: \chi}{\Xi\langle\overrightarrow{A \sqcap B}: y\rangle \Rightarrow C: \chi} \sqcap L_{2} \\
& \frac{\Xi \Rightarrow A: \chi \quad \Xi \Rightarrow B: \chi}{\Xi \Rightarrow A \sqcap B: \chi} \sqcap R
\end{aligned}
$$

cally inactive multiplicatives $\{\bullet-, \multimap, \circ, \rightarrow, \boldsymbol{\bullet}, \boldsymbol{\top}, \uparrow, \downarrow, \uparrow, \downarrow, \ominus, \ominus\}:$ Morrill and Valentín (2014b). Semantically inactive additives $\{\sqcap, \sqcup\}$ : Morrill (1994). Semantically inactive first-order quantifiers $\{\forall, \exists\}$ : Morrill (1994). Semantically inactive normal modalities $\{\boldsymbol{\square}, \boldsymbol{\forall}$ : Hepple (1990), Morrill (1994). The rules for semantically inactive variants are the same as those for the semantically active versions syntactically, but have the same label on premises and conclusions semantically; see for example Figs. 8 , 9 and $10 .{ }^{14}$

\section{Initial examples}

The first example is as follows ${ }^{15}$ :

\section{(15) $[$ john]+walks : $S f$}

Note that in our syntactical form the subject is a bracketed domain, and this will generally be the case-implementing that subjects are weak islands. Lookup in our lexicon yields the following semantically labelled sequent:

\footnotetext{
14 The synthetic connectives are: left and right projection and injection $\left\{\triangleleft^{-1}, \triangleright^{-1}, \triangleleft, \triangleright\right\}$, Morrill et al. (2009); split and bridge $\left\{{ }^{\wedge},{ }^{\wedge}\right\}$, Morrill and Merenciano (1996); continuous and discontinuous nondeterministic multiplicatives $\{\div, \otimes, \Uparrow, \Downarrow, \odot\}$, Morrill et al. (2011). The difference operator - of Morrill and Valentín (2014a) has application to linguistic exceptions.

15 The derivations we give have been computer-generated from a lexicon (given in Appendix 2) and parser CatLog2 for the categorial logic, available at http://www.cs.upc.edu/ morrill. There is no particular reason for the exact constitution of the lexicon; it exemplifies the state of experimentation at the time that the subfragment of derivations presented in this paper was generated. The implementation is a categorial parser/theorem-prover CatLog2 comprising 6000 lines of Prolog using backward chaining proof-search in the Gentzen sequent calculus (Morrill 2011a), and the focusing of Andreoli (1992); see Morrill and Valentín (2015b). In addition to focusing, the implementation exploits count-invariance (van Benthem 1991; Valentín et al. 2013). In focusing, proofs are built in alternating phases of don't care nondeterministic invertible/asynchronous rule application and focused noninvertible/synchronous rule application. The boxes in our derivations mark the focused types, which are the active types of synchronous rule application. All the reader needs to keep in mind is that if there is a boxed type in the conclusion of an inference step then it is the active type of that inference step, i.e. the type which is decomposed reading from conclusion to premises.
} 
(16)

$[\square N t(s(m)): j], \square(\langle\rangle \exists g N t(s(g)) \backslash S f):{ }^{\wedge} \lambda A(\operatorname{Pres}(\ulcorner$ walk $A)) \Rightarrow S f$

The lexical types are semantically modalised outermost, and this will always be the case-implementing that word meanings are intensions/senses; the modality of the proper name subject is semantically inactive (proper names are rigid designators), while the modality of the tensed verb is semantically active (the interpretation of tensed verbs depends on the temporal reference points). The verb projects a finite sentence (feature $f$ ) when it combines with a third person singular (bracketed) subject of any gender $g$ (the existential quantification); the actual subject is masculine (feature $m$ ).

The derivation is as follows:

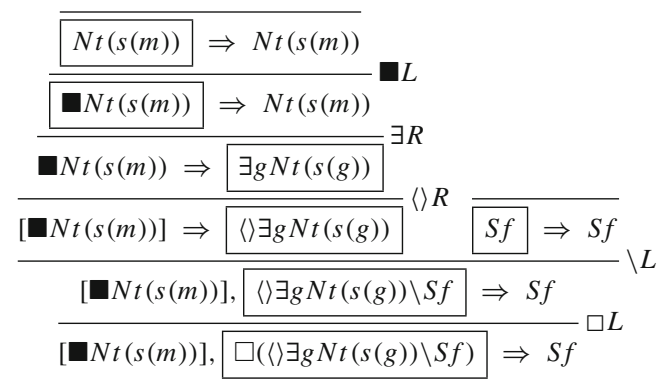

The flow of information in the semantic reading of derivations can be illustrated for the case in hand as follows; note that in practice the steps of this information flow are implemented by unification stepwise with derivation. First, variables for the antecedent semantics are added in the endsequent:

$[\square N t(s(m)): x], \square(\langle\rangle \exists g N t(s(g)) \backslash S f): y \Rightarrow S f$

Reading bottom-up, at the lowest inference step ( $\square \mathrm{L})$ the verb semantics is replaced by the extension $z$ and the subject semantics $x$ is carried over:

$$
\frac{[\square N t(s(m)): x],\langle\rangle \exists g N t(s(g)) \backslash S f: z \Rightarrow S f}{[\square N t(s(m)): x], \square(\langle\rangle \exists g N t(s(g)) \backslash S f): y \Rightarrow S f} \square L
$$

At the second inference we propagate the subject semantics on the argument branch:

$$
\frac{[\square N t(s(m)): x] \Rightarrow\langle\rangle \exists g N t(s(g)) \quad S f \Rightarrow S f}{[\square N t(s(m)): x],\langle\rangle \exists g N t(s(g)) \backslash S f: z \Rightarrow S f} \backslash L
$$

The next three inferences involve semantically transparent copying of the antecedent semantics:

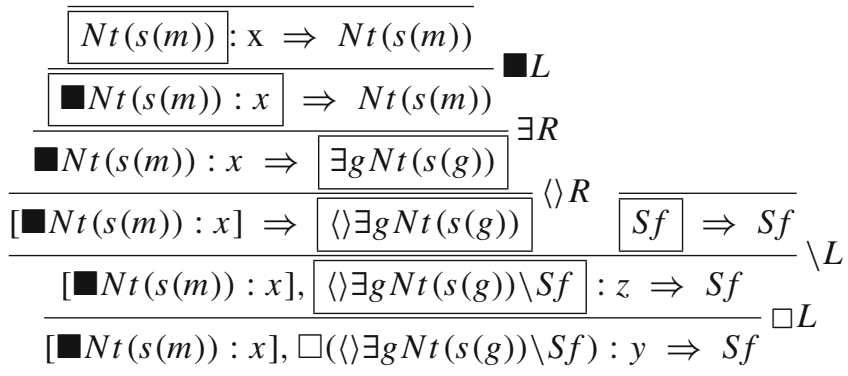


At the identity axiom the antecedent semantics is copied to the succedent:

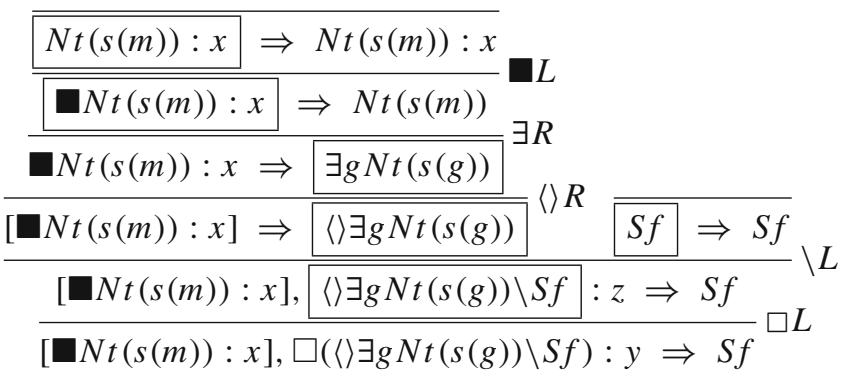

In a following phase the succedent semantics is copied from premises to conclusions as far as the root of the argument branch:

$$
\begin{aligned}
& \begin{array}{l}
\overline{N t(s(m)): x} \Rightarrow N t(s(m)): x \\
\hline \hline \mathbf{D t}(s(m)): x \Rightarrow N t(s(m)): x
\end{array} \\
& \overline{\mathbf{N} t(s(m)): x \Rightarrow \exists g N t(s(g)): x} \exists R \\
& \overline{[\mathbf{D} t(s(m)): x] \Rightarrow\langle\rangle \exists g N t(s(g)): x}\langle\rangle R \quad \overline{\quad S f \Rightarrow S f} \backslash L
\end{aligned}
$$

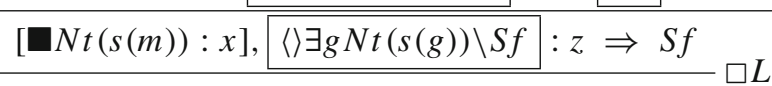

$$
\begin{aligned}
& {[\square t(s(m)): x], \square(\langle\rangle \exists g N t(s(g)) \backslash S f): y \Rightarrow S f}
\end{aligned}
$$

Now the functor value semantics in the antecedent of the value branch is labelled with a new variable $w$ :

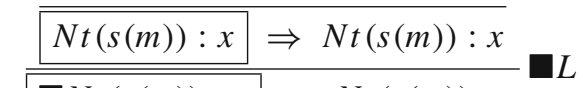

$$
\begin{aligned}
& \boldsymbol{\square} N t(s(m)): x \Rightarrow N t(s(m)): x \\
& \overline{\mathbf{N} t(s(m)): x \Rightarrow \exists g N t(s(g)): x} \exists R
\end{aligned}
$$

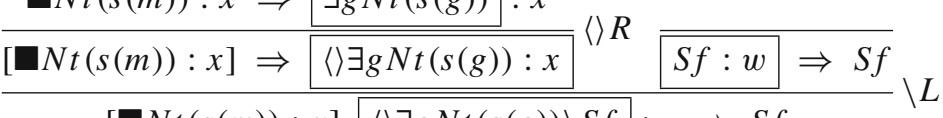

$$
\begin{aligned}
& {[\boldsymbol{\square} N t(s(m)): x],\langle\rangle \exists g N t(s(g)) \backslash S f: z \Rightarrow S f} \\
& {[\square N t(s(m)): x], \square(\langle\rangle \exists g N t(s(g)) \backslash S f): y \Rightarrow S f}
\end{aligned}
$$

At the id axiom this semantics is copied from antecedent to succedent:

$$
\begin{aligned}
& N t(s(m)): x \Rightarrow N t(s(m)): x
\end{aligned}
$$

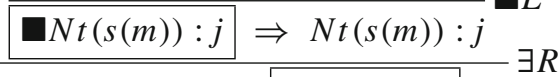

$$
\begin{aligned}
& \overline{\mathrm{N} t(s(m)): x \Rightarrow \exists g N t(s(g)): x}
\end{aligned}
$$

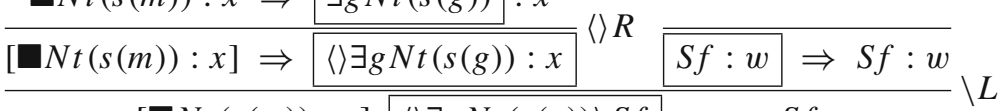

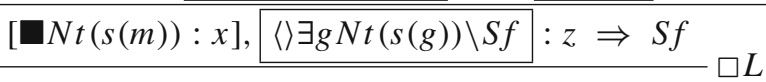

$$
\begin{aligned}
& {[\square N t(s(m)): x], \square(\langle\rangle \exists g N t(s(g)) \backslash S f): y \Rightarrow S f}
\end{aligned}
$$


In the $\backslash L$ conclusion succedent the semantics of the major premise is subject to the substitution of $w$ by the functional application of the functor $z$ to the argument $x$ :

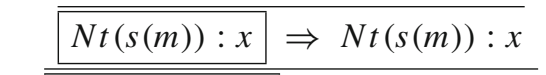

$$
\begin{aligned}
& \boldsymbol{\square} N t(s(m)): x \Rightarrow N t(s(m)): x \\
& \frac{\square N t(s(m)): x \Rightarrow \exists g N(s(g)): x}{\square R}\langle\rangle \\
& \overline{[\square N t(s(m)): x] \Rightarrow\langle\rangle \exists g N(s(g)): x}\langle\rangle R \overline{\quad S f: w \Rightarrow S f: w} \backslash L \\
& \overline{[\square t(s(m)): x],\langle\rangle \exists g N t(s(g)) \backslash S f: z \Rightarrow S f: w\{(z x) / w\}=(z x)} \backslash L \\
& {[\square N t(s(m)): x], \square(\langle\rangle \exists g N t(s(g)) \backslash S f): y \Rightarrow S f}
\end{aligned}
$$

And thence to the conclusion of the endsequent:

$$
\begin{aligned}
& \overline{N t(s(m)): x \Rightarrow N t(s(m)): x} \mathbf{L} \\
& \mathbf{\square} t(s(m)): x \Rightarrow N t(s(m)): x \\
& \overline{\operatorname{Nt}(s(m)): x \Rightarrow \exists g N t(s(g)): x} \exists R
\end{aligned}
$$

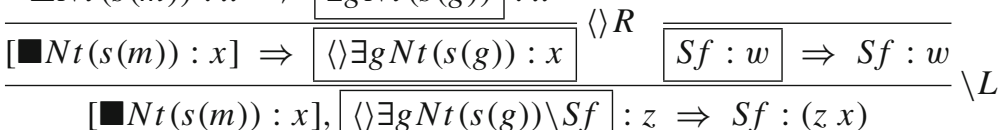

$$
\begin{aligned}
& {[\square N(s(m)): x], \square(\langle\rangle \exists g N t(s(g)) \backslash S f): y \Rightarrow S f:(z x)\{\sim y / z\}=(\ulcorner y x)}
\end{aligned}
$$

Now we can substitute in the lexical semantics $j$ for John $(x)$ and ${ }^{\wedge} \lambda A(\operatorname{Pres}(\ulcorner$ walk $A)$ ) for walks $(y)$ and evaluate: ${ }^{16}$

$$
\begin{aligned}
& \left({ }^{\wedge} \lambda A(\operatorname{Pres}(\backsim \text { walk } A)) j\right)= \\
& (\lambda A(\operatorname{Pres}(\backsim \text { walk } A)) j)= \\
& (\operatorname{Pres}(\longleftarrow \text { walk } j))
\end{aligned}
$$

(As we have said, this elucidation is not exactly how CatLog2 extracts semantics; CatLog2 uses unification and instantiation of metavariables to deliver in a single pass the unevaluated semantics of the upwards and downward phases, and then normalises.)

By way of a second example, the following is a simple transitive sentence:

(29) $[$ john] + loves + mary : $S f$

Lexical lookup yields:

$$
\begin{aligned}
& {[\square N t(s(m)): j], \square((\langle\rangle \exists g N t(s(g)) \backslash S f) / \exists a N a):{ }^{\wedge} \lambda A \lambda B\left(\text { Pres }\left(\left({ }^{\prime} \text { love } A\right) B\right)\right),} \\
& \square N t(s(f)): m \Rightarrow S f
\end{aligned}
$$

There is the derivation given in Fig. 11. Reading upwards from the endsequent, the first inference removes the intensionality modality from the transitive verb, and then over left selects the object to analyse as the argument of the transitive verb; this is

\footnotetext{
16 Montague's Intensional Logic assigned nonlogical constants of type $\tau$ a denotation in the intension of $\tau$ and then interpreted a constant with respect to a world as its extension in that world. By contrast our semantic representation language assigns constants denotations in their own type, so our semantic representations have explicit extensionalisations of intensional constants.
} 


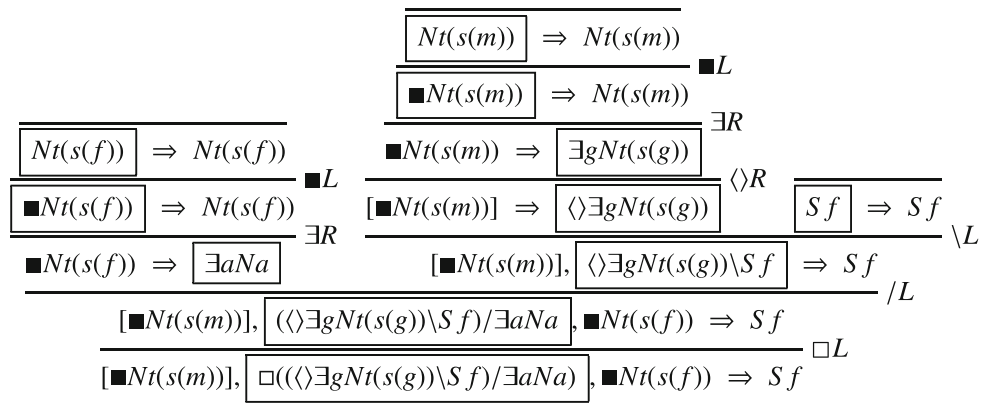

Fig. 11 Derivation for John loves Mary

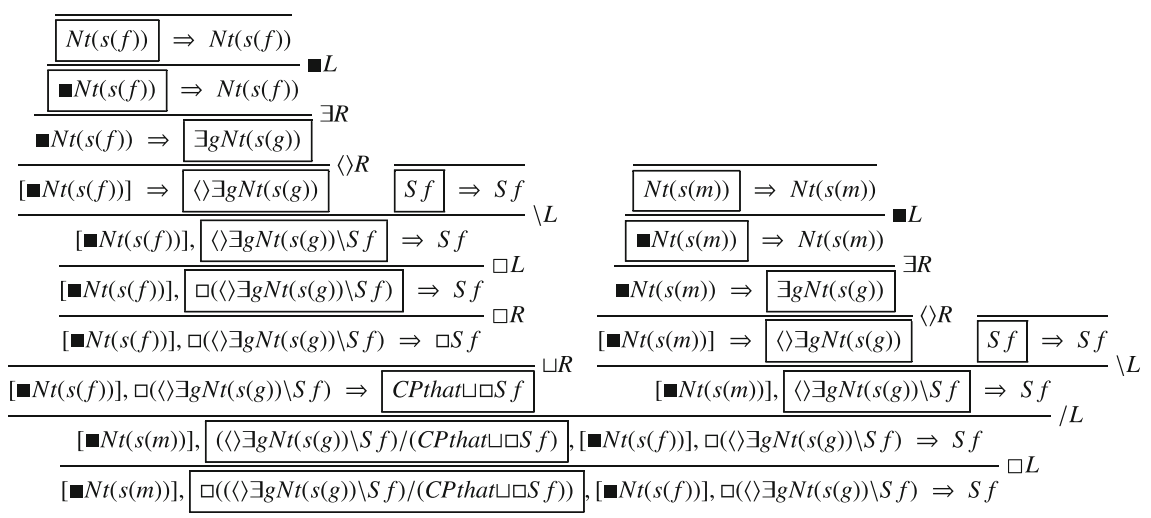

Fig. 12 Derivation for John thinks Mary walks

done by existential right instantiating the agreement feature to third person singular feminine, followed by (semantically inactive) intensionality modality left. The right hand branch is the same as for example (15) after the first inference. All this delivers semantics:

(31) (Pres $(($ love $m) j))$

The next example has a subordinate clause:

(32) $[$ john $]+$ thinks $+[$ mary $]+$ walks : $S f$

Lexical lookup yields the following; note that the propositional attitude verb is polymorphic with respect to a complementised or uncomplementised sentential argument, expressed with a semantically inactive additive disjunction:

$$
\begin{aligned}
& {[\square N t(s(m)): j], \square((\langle\rangle \exists g N t(s(g)) \backslash S f) /(C P \text { that } \sqcup \square S f)):} \\
& { }^{\lambda} \lambda A B B(\text { Pres }((\smile \text { think } A) B)),[\square N t(s(f)): m], \\
& \square(\langle\rangle \exists g N t(s(g)) \backslash S f):{ }^{\wedge} \lambda C(\text { Pres }(\longleftarrow \text { walk } C)) \Rightarrow S f
\end{aligned}
$$

This has the derivation given in Fig. 12. Reading bottom-up, following elimination of the intensionality modality of the propositional attitude verb, over left partitions in such a way as to supply the subordinate clause as the propositional argument. Again, 


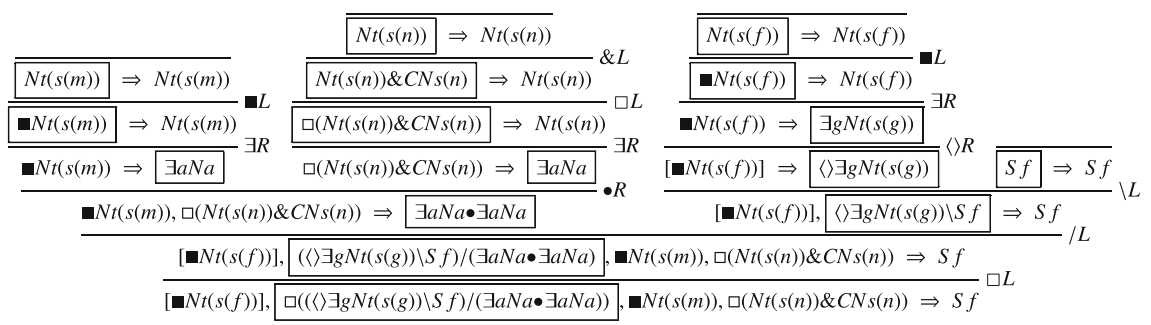

Fig. 13 Derivation for Mary buys John coffee

the righthand subtree is the same as for example (15) after the first inference. In the lefthand subtree semantically inactive additive conjunction right selects the modalised uncomplementized sentence type. The succedent modality is removed, this removal being licensed by the fact that all the antecedent types are modalised, and the remaining derivation is also like that for example (15). The derivation delivers semantics:

\section{$\left(\right.$ Pres $\left(\left({ }^{\circ}\right.\right.$ think ${ }^{\wedge}($ Pres $(\smile$ walk $\left.\left.\left.m))\right) j\right)\right)$}

The following example involves a ditransitive verb:

$$
[\text { mary }]+\text { buys }+ \text { john }+ \text { coffee }: S f
$$

Lexical lookup is as follows; note the use of (continuous) product (multiplicative conjunction) for the ditransitive verb, and the use of additive conjunction for the polymorphism of the mass noun coffee which can appear either as a bare nominal or with an article:

$$
\begin{aligned}
& {[\square N t(s(f)): m], \square((\langle\rangle \exists g N t(s(g)) \backslash S f) /(\exists a N a \bullet \exists a N a)):} \\
& { }^{\wedge} \lambda \lambda B\left(\text { Pres }\left(\left(\left({ }^{\prime} \text { buy } \pi_{1} A\right) \pi_{2} A\right) B\right)\right), \square N t(s(m)): j, \\
& \square(N t(s(n)) \& C N s(n)):{ }^{\wedge}((g e n ` c o f f e e), ` c o f f e e) \Longrightarrow S f
\end{aligned}
$$

There is the derivation given in Fig. 13. After removal of the outer modality of the ditransitive verb, the partitioning of over left selects the two objects as the verb's product argument, partitioned in turn by product right. The indirect object John is analysed by existential right and inactive modality left inferences; the direct object coffee is analysed by existential right and (active) modality left inferences followed by selection of the bare noun type by additive conjunction left. The rightmost subtree is as usual for an intransitive sentence. This delivers semantics as follows in which a 'generic' operator applies to coffee:

$$
\text { (Pres } \left.\left(\left(\left({ }^{\prime} b u y j\right)(\text { gen } ` \text { coffee })\right) m\right)\right)
$$

The next example includes a definite article:

$$
\text { (38) }[\text { the }+ \text { man }]+\text { walks : } S f
$$

We treat the definite article simply as an iota operator which returns the unique individual in the context of discourse satisfying its common noun argument (Carpenter 1997); this unicity is presupposed by the use of the definite. Lexical lookup yields the semantically labelled sequent: 


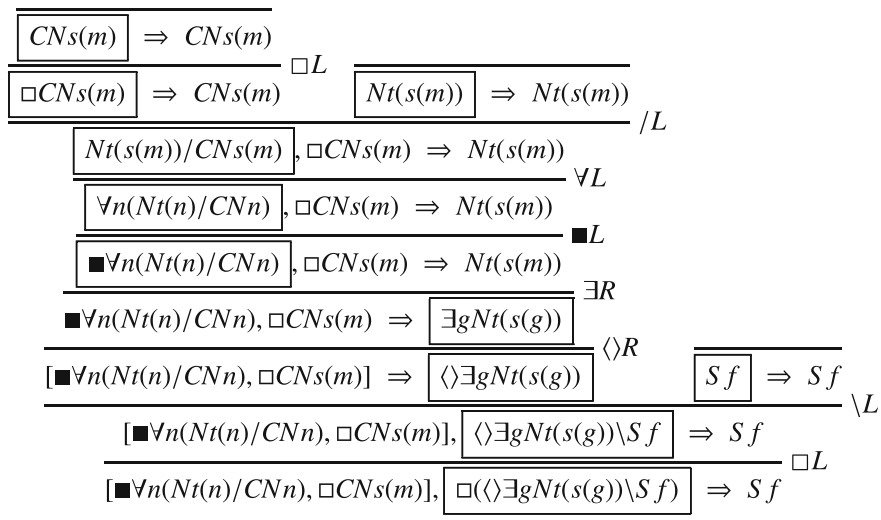

Fig. 14 Derivation for The man walks

$$
\begin{aligned}
& {[\square \forall n(N t(n) / C N n): \iota, \square C N s(m): \text { man }], \square(\langle\rangle \exists g N t(s(g)) \backslash S f):} \\
& { }_{\lambda A(\operatorname{Pres}(\longleftarrow \text { walkA })) \Rightarrow S f}^{\Rightarrow}
\end{aligned}
$$

There is the derivation given in Fig. 14. This is like the derivation of an intransitive sentence before, but with the analysis of the definite noun phrase subject at the top left. The derivation delivers semantics:

(40) $($ Pres (`walk (८`man)))

The next two examples have adverbial and adnominal prepositional modification respectively. We consider the adverbial case first:

$$
[\text { john }]+\text { walks }+ \text { from }+ \text { edinburgh }: S f
$$

Lexical lookup inserts a single value-polymorphic prepositional type, which uses semantically active additive conjunction:

$$
\begin{aligned}
& {[\square N t(s(m)): j], \square(\langle\rangle \exists g N t(s(g)) \backslash S f):{ }^{\wedge} \lambda A(\text { Pres }(\smile \text { walkA })),} \\
& \square((\forall a \forall f((\langle\rangle N a \backslash S f) \backslash(\langle\rangle N a \backslash S f)) \& \forall n(C N n \backslash C N n)) / \exists b N b): \\
& { }^{\lambda} B((\smile \text { fromadv B), }(\curlyvee \text { fromadn } B)), \square N t(s(n)): e \Rightarrow S f
\end{aligned}
$$

There is the derivation given in Fig. 15. After elimination of the outer modality of the preposition, over left selects as the prepositional argument the prepositional object, which is analysed in the leftmost subtree. In the sister subtree additive conjunction left selects the adverbial type for the prepositional phrase and for all left instantiates the subject agreement and verb form features to third person singular masculine, and finite. Following under left, in the middle subtree walks is analysed as the intransitive verb second argument of the adverbial preposition; note the analysis of the higherorder type by the under right rule, which lowers the conclusion succedent hypothetical subtype into the premise antecedent. The rightmost subtree is an intransitive sentence instance again. All this delivers the semantics:

$$
(((\text { fromadve }) \lambda B(\text { Pres }(\ulcorner\text { walk } B))) j)
$$

The adnominal case is: 


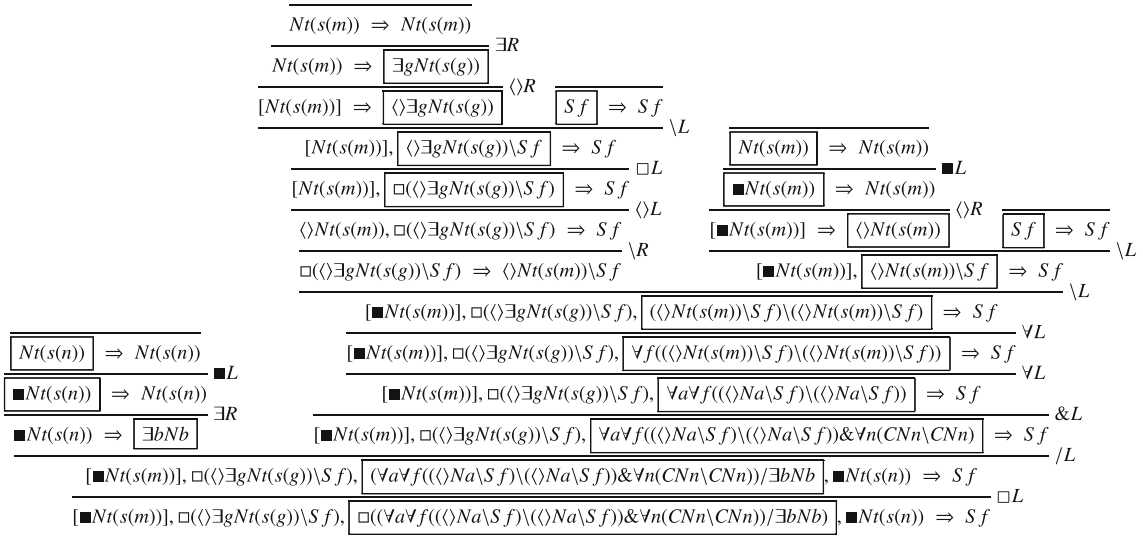

Fig. 15 Derivation for John walks from Edinburgh

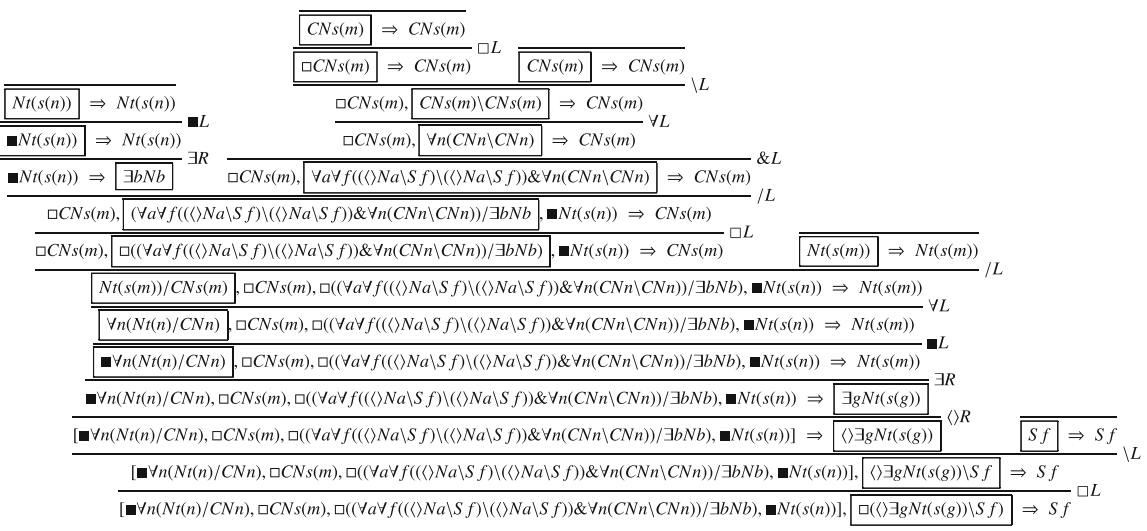

Fig. 16 Derivation for The man from Edinburgh walks

\section{(44) $[$ the + man + from + edinburgh $]+$ walks : $S f$}

Lexical lookup yields:

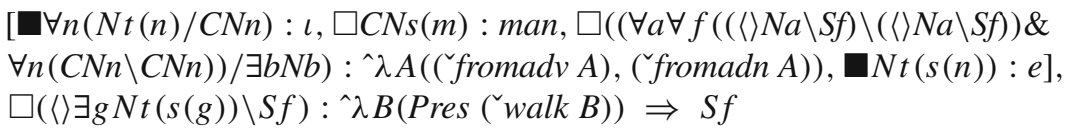

There is the derivation given in Fig. 16. In the first two steps the intransitive verb walks is prepared to apply to the complex subject. Bracket right and exists right follow, then (inactive) modality left and for all left on the determiner, which then applies to the complex common noun. The result of modality left on the preposition applies to the prepositional object and in the major premise additive conjunction left selects the adnominal prepositional type. The semantics delivered is:

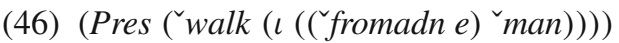

The last two initial examples involve the copula with nominal and (intersective) adjectival complementation respectively. We consider first the nominal case: 


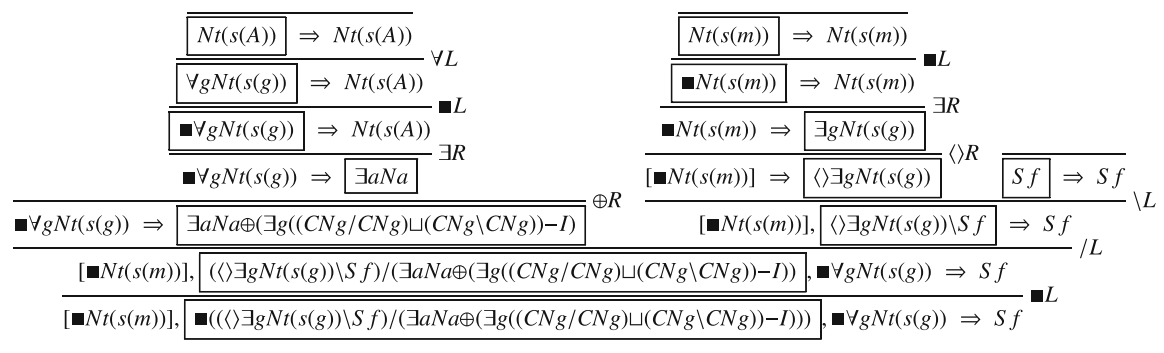

Fig. 17 Derivation for Tully is Cicero

$$
[\text { tully }]+\text { is }+ \text { cicero }: S f
$$

Lexical lookup inserts a single argument-polymorphic copula type, which uses both semantically active and semantically inactive additive disjunction: ${ }^{17}$

$$
\begin{aligned}
& {[\square N t(s(m)): t], \mathbf{\square}((\langle\exists g N t(s(g)) \backslash S f) /} \\
& (\exists a N a \oplus(\exists g((C N g / C N g) \sqcup(C N g \backslash C N g))-I))): \lambda A \lambda B(\text { Pres }(A \rightarrow C .[B=C] ; \\
& D .((D \lambda E[E=B]) B))), \square \forall g N t(s(g)): \text { cicero } \Rightarrow S f
\end{aligned}
$$

There is the derivation given in Fig. 17. After elimination of the outer copula modality the copula is applied to its nominal complement. Additive disjunction right selects the first, nominal, disjunct. The derivation delivers semantics:

$$
(\text { Pres }[t=c])
$$

The (intersective) adjectival case is:

$$
[\text { tully }]+\text { is }+ \text { humanist : } S f
$$

Lexical lookup yields:

$$
\begin{aligned}
& {[\square N t(s(m)): t], \mathbf{\square}((\langle\rangle \exists g N t(s(g)) \backslash S f) /(\exists a N a \oplus(\exists g((C N g / C N g) \sqcup} \\
& (C N g \backslash C N g))-I))): \lambda A \lambda B(P r e s(A \rightarrow C .[B=C] ; D .((D \lambda E[E=B]) B))), \\
& \square \forall n(C N n / C N n):{ }^{\wedge} \lambda F \lambda G[(F G) \wedge(\smile \text { humanist } G)] \Rightarrow S f
\end{aligned}
$$

There is the derivation given in Fig. 18. After elimination of its outer modality, the copula is applied to its adjectival complement. Semantically active additive disjunction right selects the second disjunct. The difference right rule checks that the antecedent is not empty, but this is not displayed. Exists right substitutes the existentially quantified variable for a metavariable $A$ and semantically inactive additive disjunction right then selects the adjectival disjunct. The following semantics is delivered:

(52) (Pres (`humanist $t)$ )

\footnotetext{
17 The difference operator (Morrill and Valentín 2014a) for linguistic exceptions is also used. It involves negation as failure, which cannot easily be displayed. We do not dwell on this operator here.
} 


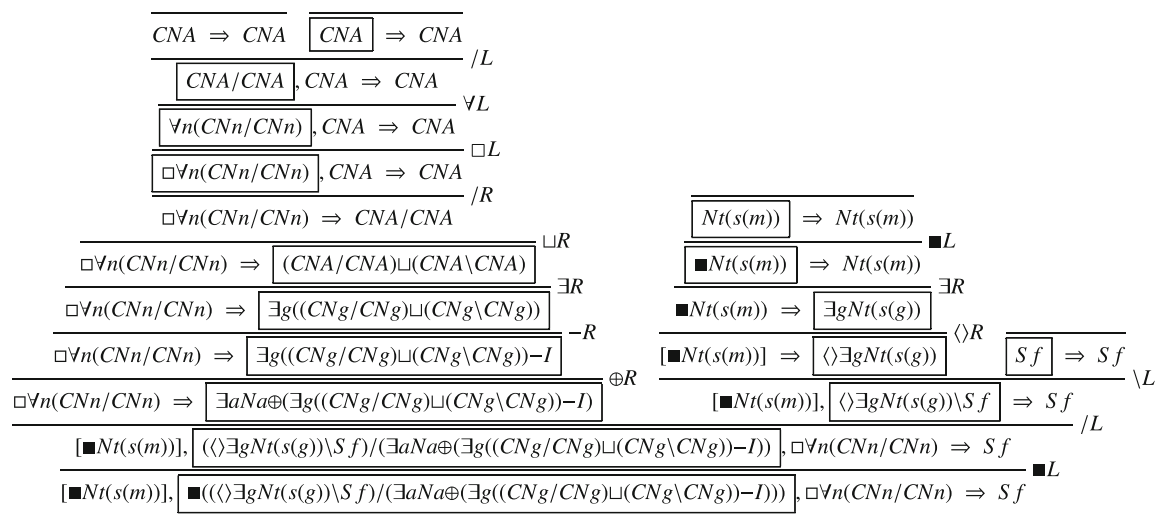

Fig. 18 Derivation for Tully is humanist

\section{Routes we do not take}

Szabolcsi (1983) and Steedman (1987) aim to account for parasitic gaps in combinatory categorial grammar (CCG) by means of the combinator $\mathbf{S}$ such that $\mathbf{S} x y z=$ $\left(\begin{array}{l}x z \\ )\end{array}\right)(y z)$, for example positing a combinatory schema:

$$
y: Y / Z, x:(Y \backslash X) / Z \Rightarrow \mathbf{S} x \quad y: X / Z
$$

Such a schema makes no sense from the point of view of the logicisation of grammar pursued here. The rule is not Lambek-valid and any semantics validating it would also validate schemata which overgenerate massively. So much the worse, the proponents of CCG would say, for grammar as logic: grammar is a formal system but not a logic, and one should not care about things like soundness and completeness.

CCG and type logical grammar agree on the task of defining syntax and semantics of the (object) natural language. What is curious about CCG is that at the same time it declines to consider syntax (proof theory) and semantics (model theory) of the (meta-)linguistic formalism. A CCG account of parasitic gaps, which employs just the directional slashes and a minimum of combinatory schemata, must capture the effects of structural inhibition (islands) and structural facilitation (parasiticy) by good fortune in the interaction of the combinatory schemata chosen and the categorial types occurring in grammar. In our approach control of structural inhibition by bracket modalities and control of structural facilitation by exponentials are separated in an analysis recognising the distinct algebraic roles of variation from an associative and linear regime. This type logical approach lets us state our analysis with clarity in the knowledge that whatever the empirical adequacy, metatheoretical facts are known. In CCG the metatheory is not logically investigated because it is not formulated logically.

It is interesting to ask why we treat medial extraction here with ! rather than with $\uparrow$ as illustrated in Sect. 2 (cf. also Moortgat 1988; Muskens 2003; Mihaliček and Pollard 2012; Barker and Shan 2015; and Kubota and Levine 2015). The answer is that, on the one hand, $\uparrow$ as defined does not respect island constraints and, on the other hand, $\uparrow$ does not extend to parasitic gaps: it is unclear how a single local inference rule can account for unbounded recursive nesting of parasitic gaps in subislands. Our treatment 
in terms of ! both respects islands, and extends to (unbounded numbers of) parasitic gaps through iteration of contraction. ${ }^{18}$

An option available in both CCG and type logical grammar is to attempt to analyse the nonlinearity of parasitic extraction not syntactically but lexically. Thus for example Jansche and Vasishth (2002) propose induction of parasitic gaps in adverbial clauses by a lexicalised gap-duplicating effect in the adverbial head. All contexts allowing parasitic gaps would require a corresponding gap-duplicating lexical ambiguity. The appeal to lexical ambiguity in lexical grammar formalisms is as frequent as it is untenable. Every ambiguity of every item doubles the lexical insertion search space. And in the case in hand there is to our knowledge no independent evidence, such as difference in meaning, for lexical ambiguity underlying parasitic extraction. We continue on the assumption that it is indeed a syntactic phenomenon.

\section{Relativisation}

Our account of relativisation rests on the lexical projection of islands by argument bracketing $(\langle\rangle)$ and value antibracketing $\left([]^{-1}\right)$, and a single relative pronoun type of overall shape $R /((\langle\rangle N \sqcap ! N) \backslash S)$ for both subject and object relativisation. Note that the two operands of the hypothetical gap subtype are conjoined because in analysis of the body of relative clauses the higher order succedent argument of form \langle\rangle $N \sqcap$ !N is lowered into the antecedent according to the deduction theorem, where the conjunction left rule selects the first or second operand to produce the subject/object relativisation alternation.

In subject relativisation \langle\rangle $N$ is selected by conjunction left, and satisfies the (bracketed) subject valency.

In object relativisation $! N$ is selected by conjunction left; when the $! L$ rule is applied to $! N$, the hypothetical subtype $N$ moves into the stoup, from whence it can move by $! P$ to any (nonisland) position in its zone, realising nonparasitic extraction. However, in addition it can be copied by $! C$ to the stoup of a newly created weak island domain, realising parasitic extraction. The $N$ in the outer stoop can be copied by $C C$ repeatedly, capturing that there may be parasitic gaps in any number of local weak islands; at the end of this process it moves by $! P$ to a host position in its zone. The $N$ in an inner stoup can also be copied by $! C$ to the stoup of any number of newly created weak subislands, and so on recursively, capturing that parasitic gaps can also be hosts to further parasitic gaps; finally the stoup contents are copied by $! P$ to extraction sites in their zone.

In this section we analyse examples illustrating the account of relativisation. The first example is a minimal subject relativisation; note that the relative clause is doubly bracketed, corresponding to the fact that relative clauses are strong islands. ${ }^{19}$

$$
\text { man }+[[\text { that }+ \text { walks }]]: C N s(m)
$$

\footnotetext{
18 We note that the discontinuity operators serve to account for the pied-piping aspect of relativisation (see e.g. Morrill et al. 2011), though we do not go into that here.

19 As we will see relative clauses themselves, being doubly bracketed, will not allow parasitic gaps.
} 


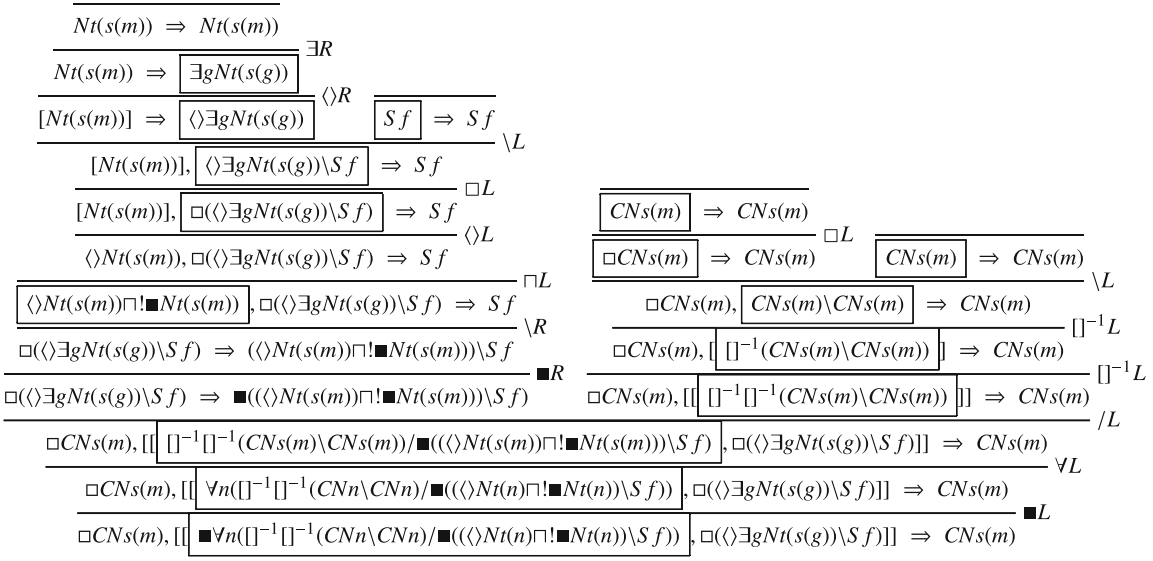

Fig. 19 Derivation for man that walks

Lexical lookup yields the following, where there is semantically inactive additive conjunction of the hypothetical subtypes \langle\rangle $N$ for subject relativisation and $! \boldsymbol{\square}$ for object relativisation; the (semantically inactive) modality on the object gap subtype is to permit object relativisation from embedded modal/intensional domains: ${ }^{20}$

$$
\begin{aligned}
& \square C N s(m): \text { man, }\left[\left[\square \forall n\left([]^{-1}[]^{-1}(C N n \backslash C N n) / \square((\langle\rangle N t(n) \sqcap ! \square N t(n)) \backslash S f)\right):\right.\right. \\
& \lambda A \lambda B \lambda C[(B C) \wedge(A C)], \square(\langle\rangle \exists g N t(s(g)) \backslash S f):{ }^{\wedge} \lambda D(\text { Pres }(\ulcorner\text { walk D) })]] \Rightarrow \\
& C N s(m)
\end{aligned}
$$

There is the derivation in Fig. 19, which starts with the relative clause doubly bracketed (this will always be the case for relativisation). After elimination of the outer (semantically inactive) modality of the relative pronoun, universal left instantiates it to agree with masculine singular. Then $/ L$ partitions in such a way as to select the intransitive verb body of the relative clause as argument of the relative pronoun. In the righthand, value, subtree two antibracket eliminations cancel the double brackets before the head common noun is modified. In the lefthand, argument, subtree (inactive) box right is enabled since the antecedent is modalised, and under right then lowers the additively conjoined hypothetical subtypes into the antecedent. Observe how in the lefthand subtree $\sqcap \mathrm{L}$ selects the subject relativisation hypothetical subtype \langle\rangle $N t(s(m))$; the remaining subderivation is the usual intransitive sentence analysis. This delivers the required semantics:

(56) $\lambda C[(\ulcorner$ man $C) \wedge(\operatorname{Pres}(\longleftarrow$ walk $C))]$

\footnotetext{
20 The body of the relative clause is marked as a (semantically inactive) modal domain in order to make it a scope island. This account of relative clauses as scope islands operates on essentially the same lines as the capture of Principle A by modalities (see Morrill 1990b). Thus where, say, everyone has a type $\mathbf{\square}((S \uparrow N) \downarrow S)$ the unmodalised hypothetical subtype $N$ cannot be bound outside the modal domain of the body of a relative clause in which everyone occurs. To make the $\mathbf{Q} R$ inference for the body of the relative clause, every antecedent type must be modalised. Thus if the body contains for example everyone where it is $\mathbf{\square}((S \uparrow N) \downarrow S)$ this can scope clause-internally by decomposing after the $\boldsymbol{\nabla}$ inference, but if we try to decompose it before to make it scope clause-externally it will leave the hypothetical subtype $N$ which is not modalised and so the $\boldsymbol{Q} R$ inference is blocked.
} 
The next sentence contains a minimal example of object relativisation:

$$
[\text { the }+ \text { man }+[[\text { that }+[\text { mary }]+\text { loves }]]]+\text { walks : } S f
$$

Lexical lookup yields:

$$
\begin{aligned}
& {\left[\square \forall n(N t(n) / C N n): \iota, \square C N s(m): \operatorname{man},\left[\left[\square \forall n \left([]^{-1}[]^{-1}(C N n \backslash C N n) /\right.\right.\right.\right.} \\
& \mathbf{\square}((\langle\rangle N t(n) \sqcap ! \mathbf{\square} N(n)) \backslash S f)): \lambda A \lambda B \lambda C[(B C) \wedge(A C)],[\mathbf{\square} N t(s(f)): m] \text {, } \\
& \left.\left.\left.\square((\langle\rangle \exists g N t(s(g)) \backslash S f) / \exists a N a):{ }^{\wedge} \lambda D \lambda E\left(\text { Pres }\left(\left({ }^{\prime} \text { love } D\right) E\right)\right)\right]\right]\right] \text {, } \\
& \square(\langle\rangle \exists g N t(s(g)) \backslash S f):{ }^{\wedge} \lambda F\left(\text { Pres }\left({ }^{\prime} \text { walk } F\right)\right) \Rightarrow S f
\end{aligned}
$$

There is the derivation given in Fig. 20. The lowest four inferences prepare the subject of the intransitive matrix verb and the next three prepare the relative clause modification itself, argument to the subject definite article. The analysis of the complex common noun phrase starts in the minor premise of the lowest / $L$ with (semantically inactive) modality left, and $\forall L$ instantiating agreement to masculine singular. At the middle $/ L$, the righthand subtree cancels the double brackets with the relative pronoun value antibrackets and the lefthand subtree selects the body of the relative clause as the semantically inactive modalised higher-order subject-and-object polymorphic relative pronoun argument type. After (semantically inactive) modality right, licensed since the antecedent types are modalised, the conjoined hypothetical subject is lowered by $\backslash R$ into the antecedent. Observe how $\sqcap L$ selects the object relativisation hypothetical subtype ! $N t(s(m))$ and how this subsequently percolates in the stoup, passing in particular into the minor premise branch of the upper $/ L$ inference and hence satisfying the object valency of the transitive verb love; subject and intransitive verb phrase are analysed as usual. This delivers the required semantics:

$($ Pres $(\ulcorner$ walk $(\iota \lambda D[(\ulcorner$ man $D) \wedge($ Pres $((\ulcorner$ love $D) m))])))$

An example with longer-distance object relativisation, in the context of an entire sentence, is:

(60) $[$ the + man $+[[$ that $+[$ john $]+$ thinks $+[$ mary $]+$ loves $]]]+$ walks $: S f$

Lexical lookup yields the following; note how the propositional attitude verb is polymorphic between a complementised and an uncomplementised sentential argument, expressed with a semantically inactive additive disjunction:

$$
\begin{aligned}
& {\left[\square \forall n ( N t ( n ) / C N n ) : \iota , \square C N s ( m ) : \text { man, } \left[\left[\square \forall n \left([]^{-1}[]^{-1}(C N n \backslash C N n) /\right.\right.\right.\right.} \\
& \square((\langle\rangle N t(n) \sqcap ! \square N t(n)) \backslash S f)): \lambda A \lambda B \lambda C[(B C) \wedge(A C)],[\square N t(s(m)): j], \\
& \square((\langle\rangle \exists N t(s(g)) \backslash S f) /(C P t h a t \sqcup \square S f)):{ }^{\wedge} \lambda D \lambda E(\text { Pres }((\smile \text { think } D) E)), \\
& \left.\left.[\square N t(s(f)): m], \square((\langle\rangle \exists g N t(s(g)) \backslash S f) / \exists a N a):{ }^{\wedge} \lambda F \lambda G(\text { Pres }((\smile \text { love } F) G))\right]\right], \\
& \square(\langle\rangle g N t(s(g)) \backslash S f):{ }^{\wedge} \lambda H(\text { Pres }(\smile \text { walk } H)) \Rightarrow S f
\end{aligned}
$$

There is the derivation given in Fig. 21. Inference up as far as (1) brings us to analysis of the complex common noun phrase in the lefthand subtree. The following preparation of the relative pronoun and double bracket cancellation of its value are as usual. After modality right and under right on the relative pronoun higher-order argument, $\sqcap L$ selects the object relativisation hypothetical subtype and $! L$ moves this into the stoup. In the stoup it percolates to the subordinate clause, (observe how $\sqcup R$ selects the uncomplementised sentential argument type of the propositional attitude verb) and there $! P$ moves it into position to satisfy the embedded clause object valency.

This delivers the correct semantics: 


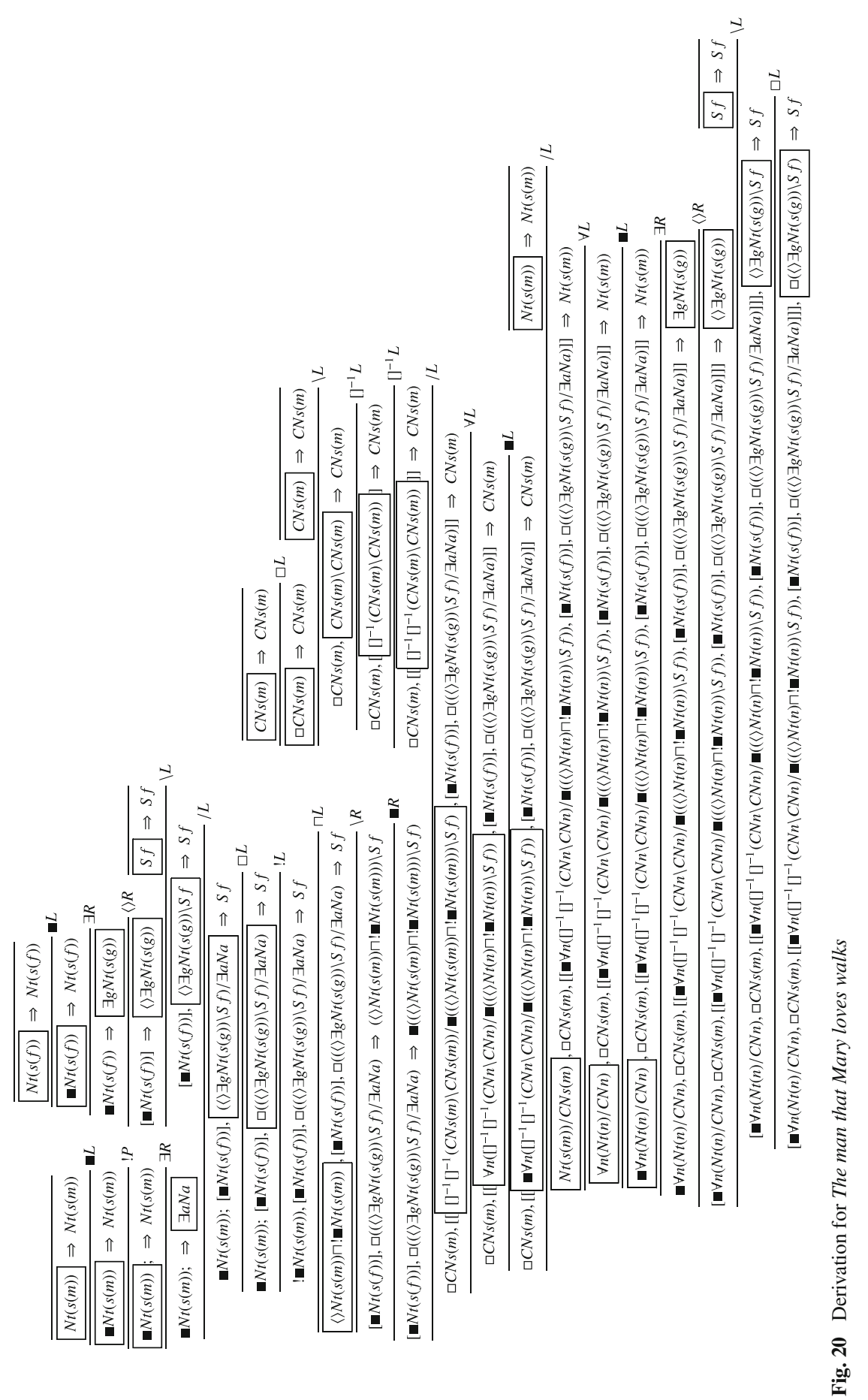




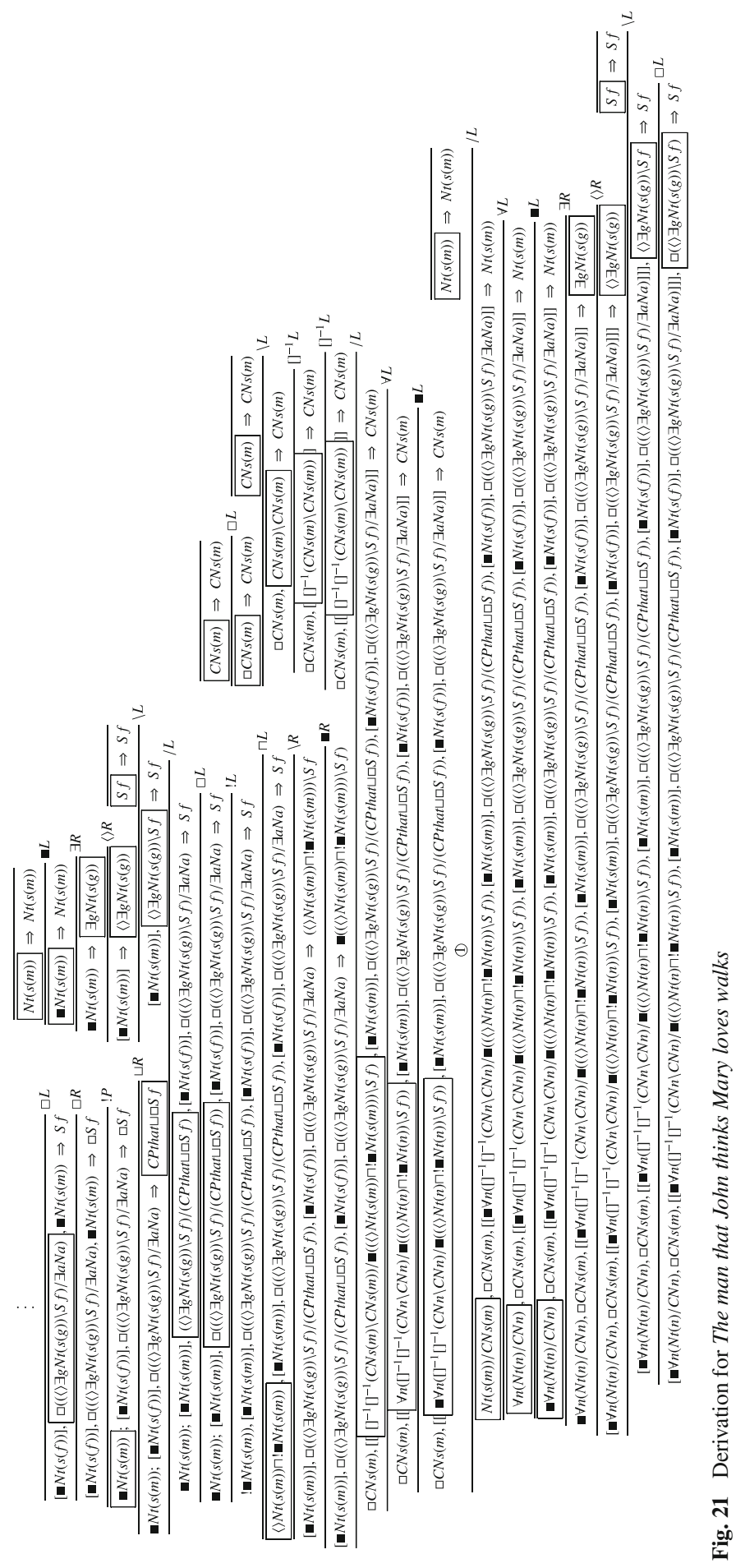




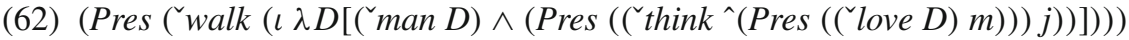

There follows an example of medial object relativisation (the gap is in a nonperipheral position left of the adverb):

(63) $\operatorname{man}+[[$ that $+[$ mary $]+$ likes + today $]]: C N s(m)$

Appropriate lexical lookup yields:

(64) $\square C N s(m):$ man, $\left[\left[\square \forall n\left([]^{-1}[]^{-1}(C N n \backslash C N n) / \square((\langle\rangle N(n) \sqcap ! \square N t(n)) \backslash S f)\right)\right.\right.$ :

$\lambda A \lambda B \lambda C[(B C) \wedge(A C)],[\square N t(s(f)): m], \square((\langle\rangle \exists g N t(s(g)) \backslash S f) / \exists a N a):$

${ }^{\wedge} \lambda D \lambda E$ (Pres (( like D) E)), $\square \forall a \forall f((\langle\rangle N a \backslash S f) \backslash(\langle\rangle N a \backslash S f))$ :

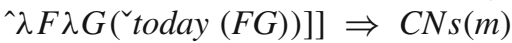

There is the derivation in Fig. 22. Analysis of the complex common noun phrase begins at the lefthand subtree (1). After modality right and conditionalisation of the conjoined hypothetical subtype, additive conjunction left applies to this latter to select the object relativisation subtype, which then moves into the stoup. After preparation of the adverb the stoup contents pass into its argument subbranch. Note how the object relativisation hypothetical gap subtype percolates in the stoup to satisfy the transitive verb object valency.

The semantics delivered is:

\section{(65) $\lambda C\left[(\smile\right.$ man $C) \wedge\left({ }^{2}\right.$ today $($ Pres $((\smile$ like $\left.\left.C) m))\right)\right]$}

As we remarked at the beginning of Sect. 3 subjects are weak islands (the Subject Condition of Chomsky 1973); accordingly in our CatLog2 fragment there is no derivation of simple relativisation from a subject such as:

(66) $\operatorname{man}+[[$ that $+[$ the + friends + of $]+$ walk $]]: C N s(m)$

This is because walk projects brackets around its subject, but the permutation of the ! hypothetical gap subtype issued by the relative pronoun is limited to its zone and cannot penetrate a bracketed subzone. Roughly, the derivation blocks at $*$ in:

$$
\frac{\frac{[N / C N, C N / P P, P P / N, N], N \backslash S \Rightarrow S}{N ;[N / C N, C N / P P, P P / N], N \backslash S \Rightarrow S}}{\frac{! N,[N / C N, C N / P P, P P / N], N \backslash S \Rightarrow S}{[N / C N, C N / P P, P P / N], N \backslash S \Rightarrow ! N \backslash S} \backslash R}
$$

However, a weak island 'parasitic' gap can be licensed by a host gap:

(68) $\mathbf{m a n}+[[$ that + the + friends + of + admire $]]: C N s(m)$

Lexical lookup yields: ${ }^{21}$

(69) $\square C N s(m):$ man, $\left[\left[\mathbf{\square} \forall n\left([]^{-1}[]^{-1}(C N n \backslash C N n) / \mathbf{\square}((\langle\rangle N t(n) \sqcap ! \square N t(n)) \backslash S f)\right)\right.\right.$ :

$\lambda A \lambda B \lambda C[(B C) \wedge(A C)], \square \forall n(N t(n) / C N n): \iota, \square(C N p / P P o f):$ friends,

$\square((\forall n(C N n \backslash C N n) / \square \exists b N b) \&(P P o f / \exists a N a)):{ }^{\wedge}\left({ }^{\sim} o f, \lambda D D\right)$,

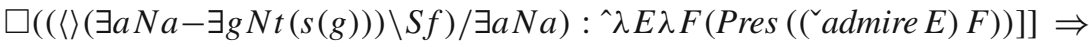
CNs $(m)$

\footnotetext{
21 We gloss over the use of 'difference' here to mark non-third person singular; its use depends on absence of derivability (negation as failure) which of course cannot easily be displayed.
} 


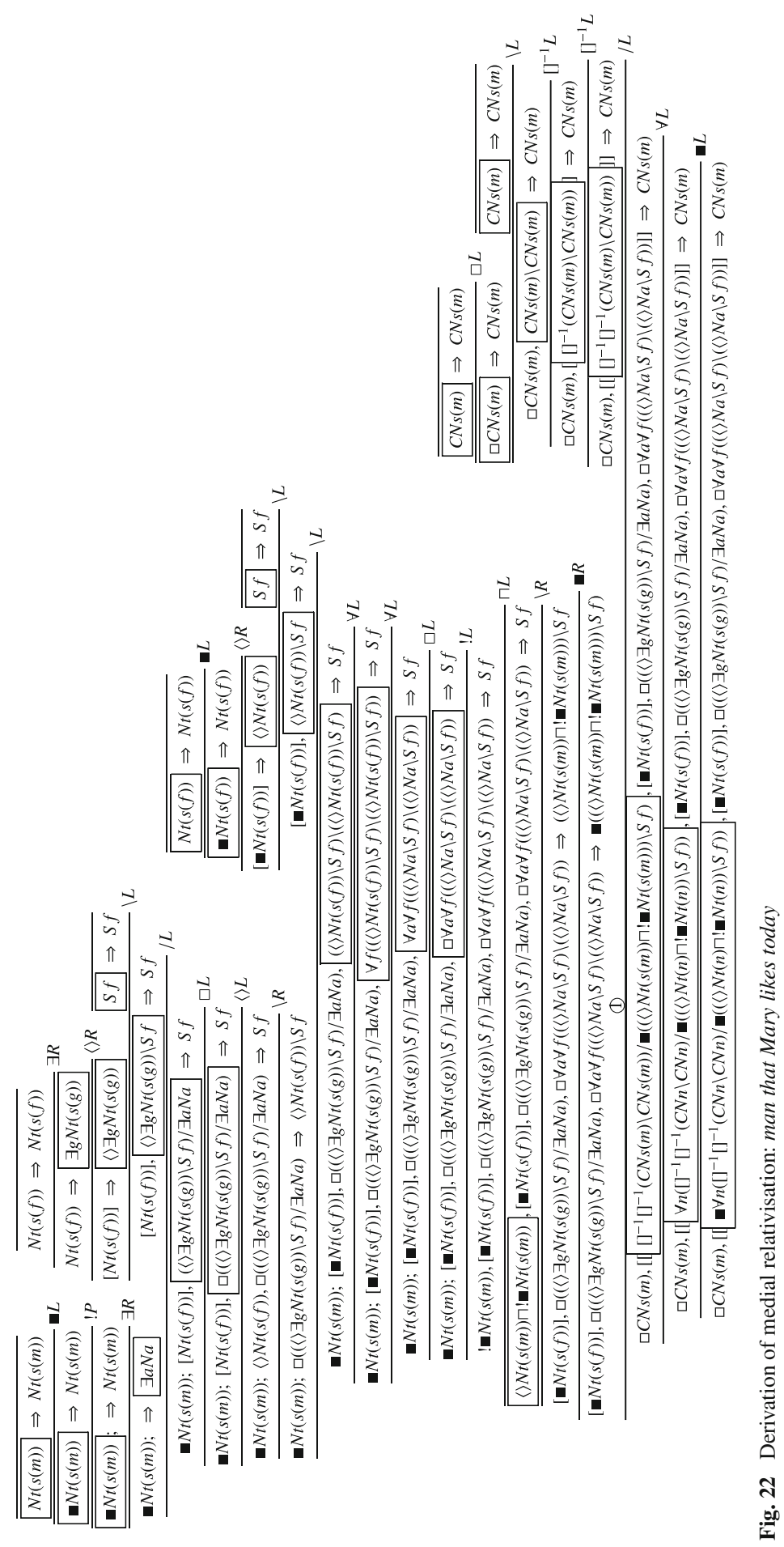


There is the derivation given in Fig. 23, where the use of contraction $! C$, involving brackets and stoups, corresponds to generating the parasitic gap. The object relativisation hypothetical subtype moves into the stoup at depth seven in the lefthand subtree (before this the analysis is standard). Contraction then applies copying the gap type into the stoup of a newly created bracketed domain around the subordinate subject. Applications of $! P$ then move the stoup contents into the object position of admire (host) and of (parasitic). This delivers the following semantics in which the gap variable is multiply bound:

\section{(70) $\lambda C[(\smile$ man $C) \wedge($ Pres $((\smile$ admire $C)(\iota($ friends $C))))]$}

Parasitic extraction from strong islands such as coordinate structures is not acceptable:

(71) * that ${ }_{i}$ Mary showed [[John and the friends of $\left.\left.t_{i}\right]\right]$ to $t_{i}$

This is successfully blocked because strong islands are doubly bracketed. Although contraction could apply twice to introduce two bracketings, a copy of the hypothetical gap subtype would remain trapped in the stoup at the intermediate level of bracketing, blocking overall derivation. Likewise, as we remarked in footnote 19, parasitic extraction is not possible from relative clauses themselves, for the same reason: a superfluous gap subtype would remain trapped in between the double brackets required for the strong island.

A parasitic gap can also appear in an adverbial weak island:

(72) paper $+[[$ that $+[$ john $]+$ filed + without + reading $]]: C N s(n)$

Lexical lookup for this example yields:

$$
\begin{aligned}
& \square C N s(n): \text { paper, }\left[\left[\square \forall n\left([]^{-1}[]^{-1}(C N n \backslash C N n) / \square((\langle\rangle N t(n) \sqcap ! \square N t(n)) \backslash S f)\right):\right.\right. \\
& \lambda A \lambda B \lambda C[(B C) \wedge(A C)],[\square N t(s(m)): j], \square((\langle\rangle \exists g N t(s(g)) \backslash S f) / \exists a N a): \\
& \wedge \operatorname{DD} \boldsymbol{E} \text { (Past (( file D) E)), } \square \forall a \forall f\left([]^{-1}((\langle\rangle N a \backslash S f) \backslash(\langle\rangle N a \backslash S f)) /(\langle\rangle N a \backslash S p s p)\right) \text { : } \\
& \left.\left.\lambda F \lambda G \lambda H[(G H) \wedge \neg(F H)], \square((\langle\rangle \exists a N a \backslash S p s p) / \exists a N a):{ }^{\wedge} \lambda I \lambda J\left(\left({ }^{2} \operatorname{read} I\right) J\right)\right]\right] \Rightarrow \\
& \text { CNs }(n)
\end{aligned}
$$

There is the derivation given in Fig. 24. This time at depth eight contraction copies the host stoup gap into the stoup of a newly created bracketed domain around the subordinate adverbial phrase. This delivers semantics:

$$
\lambda C\left[\left({ }^{2} \text { paper } C\right) \wedge[(\text { Past }((\ulcorner\text { file } C) j)) \wedge \neg((\text { read } C) j)]]\right.
$$

In our final relativisation example the host gap licences two parasitic gaps, in the subject noun phrase and in an adverbial phrase:

$$
\text { paper }+[[\text { that }+ \text { the }+ \text { editor }+ \text { of }+ \text { filed }+ \text { without }+ \text { reading }]]: C N s(n)
$$

Lexical lookup yields:

$$
\begin{aligned}
& \square C N s(n): \text { paper, }\left[\left[\square \forall n\left([]^{-1}[]^{-1}(C N n \backslash C N n) / \square((\langle\rangle N t(n) \sqcap ! \square N t(n)) \backslash S f)\right):\right.\right. \\
& \lambda A \lambda B \lambda C[(B C) \wedge(A C)], \square \forall n(N t(n) / C N n): \iota, \square(\forall g C N s(g) / P P o f): \text { editor, } \\
& \square((\forall n(C N n \backslash C N n) / \square \exists b N b) \&(P P o f / \exists a N a)):{ }^{\wedge}(\curlyvee o f, \lambda D D) \text {, } \\
& \square((\langle\rangle \exists g N t(s(g)) \backslash S f) / \exists a N a):{ }^{\wedge} \lambda E \lambda F(\text { Past (( }(\text { file E) } F)) \text {, } \\
& \square \forall a \forall f\left([]^{-1}((\langle\rangle N a \backslash S f) \backslash(\langle\rangle N a \backslash S f)) /(\langle\rangle N a \backslash S p s p)\right): \lambda G \lambda H \lambda I[(H I) \wedge \neg(G I)] \text {, } \\
& \left.\left.\square((\langle\rangle \exists a N a \backslash S p s p) / \exists a N a):{ }^{\wedge} \lambda J \lambda K\left(\left({ }^{2} \operatorname{read} J\right) K\right)\right]\right] \Rightarrow C N s(n)
\end{aligned}
$$




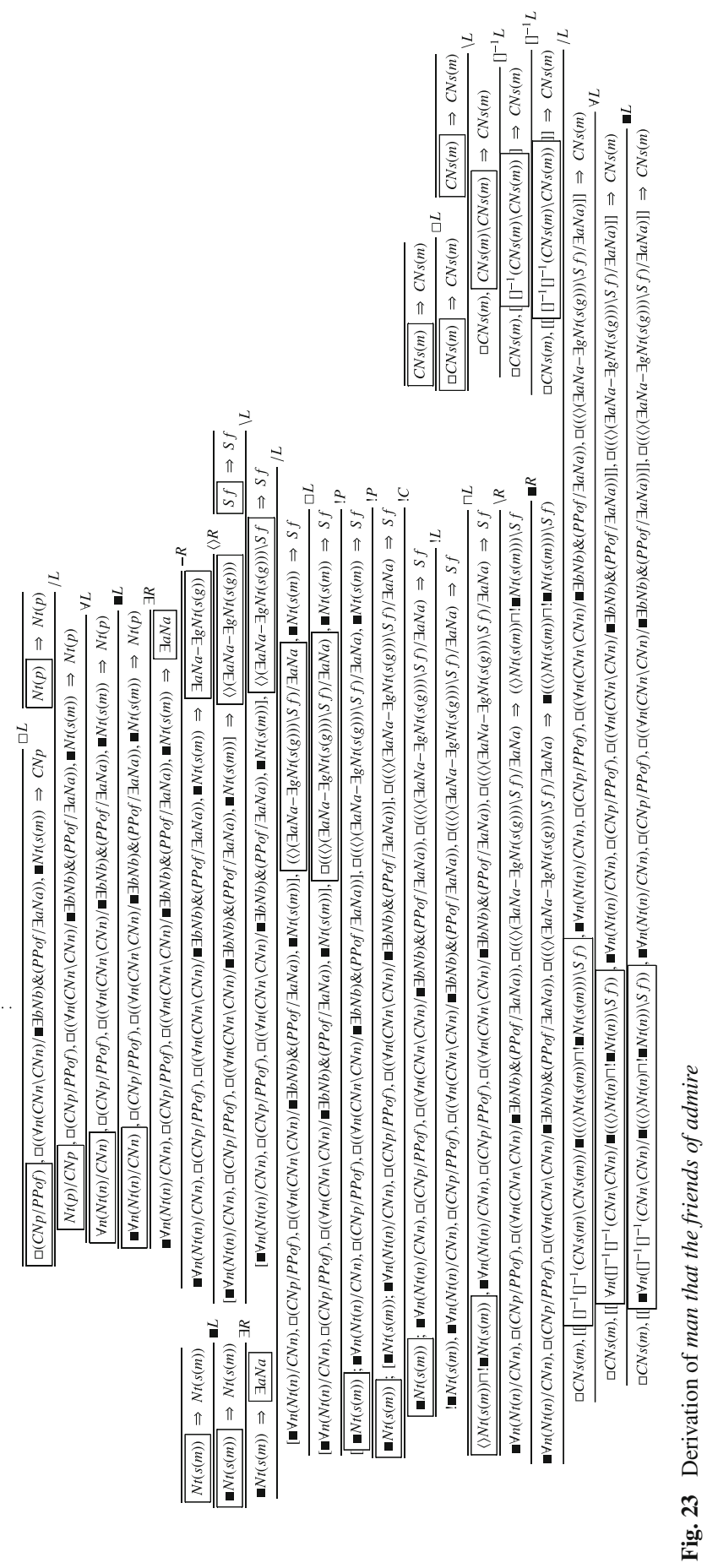




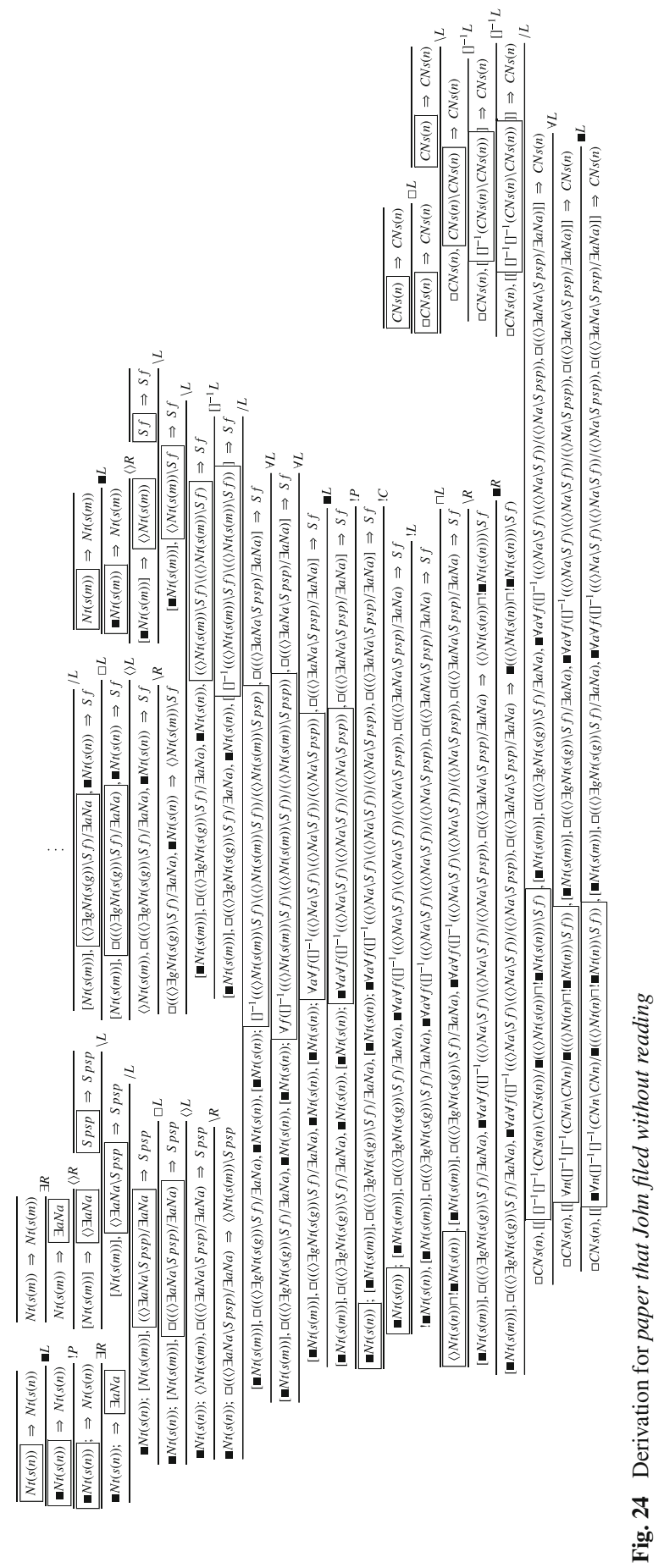


There is the derivation fragmented into Figs. 25 and 26. There are two applications of contraction, at depth nine and ten, projecting brackets around the subordinate subject and adverbial phrase and giving rise to two parasitic gaps. This delivers the correct semantics:

$$
\lambda C[(\ulcorner\text { paper } C) \wedge[(\text { Past }((\ulcorner\text { file } C)(\iota(\ulcorner\text { editor } C)))) \wedge \neg((\text { read } C)(\iota(\ulcorner\text { editor } C)))]]
$$

By now we take it that the principles of generation of recursively nested parasitic gaps are also clear.

\section{Possible exceptions}

In this section we address three kinds of possible exceptions to the account given here, along lines anticipated in the introduction.

Firstly, there are examples in which there appears to be a parasitic gap which is not in an island. The following is example (8a) from Postal (1993):

(78) man $w_{h o}$ Mary convinced $t_{i}$ that John wanted to visit $t_{i}$

And an anonymous referee points out:

(79) people whom $i$ you sent pictures of $t_{i}$ to $t_{i}$

In respect of such examples we have suggested that although there seems to be no island, there could be one. This is effected as follows for (78). Instead of a type of the form $((N \backslash S) / C P) / N$ for convince we assume $((N \backslash S) / C P) /(N \sqcup\langle\rangle N)$ where the semantically inactive additive disjunction disjunct $N$ will be selected ordinarily, and \langle\rangle $N$ when there is parasitic extraction, as in (78). Similarly for (79) we assume for picture type $(C N / P P) /(P P \sqcup\langle\rangle P P)$ where the second disjunct projects the brackets of a weak island. ${ }^{22}$ Thus in examples such as the following the semantically inactive additive disjunction inference for convince of type $((N \backslash S) / C P) /(N \sqcup\langle\rangle N)$ will select $N$ :

(80) a. man who ${ }_{i}$ Mary convinced $t_{i}$ that John wanted to visit Suzy

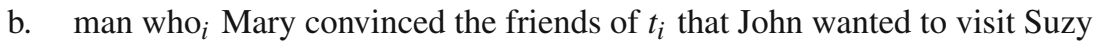

But for (78) the semantically inactive additive disjunction inference for convince of type $((N \backslash S) / C P) /(N \sqcup\langle\rangle N)$ will select \langle\rangle $N$. Similarly for the picture noun case (79).

Secondly, recall the example (4) man who a friend of laughed of escape from an indefinite subject island. The standard type for an indefinite would be of the form $((S \uparrow N) \downarrow S) / C N$, but if the type were $((S \uparrow\langle\rangle N) \downarrow S) / C N$ the example (4) would be generated since the \langle\rangle $N$ hypothetical subtype will satisfy the bracketed subject valency without any input brackets blocking the extraction of the object of the preposition. The effect of the standard type is still required to satisfy valencies such as objects which are

\footnotetext{
22 The argument pattern $X \sqcup\langle\rangle X$ is a general mechanism for an argument optional island $X$. Likewise the dual value pattern $X \sqcap[]^{-1} X$ is a general mechanism for a value optional island $X$. We could define synthetic connectives $(\langle\rangle)_{\sqcup} X=X \sqcup\langle\rangle X,(\langle\rangle)_{\sqcap} X=X \sqcap\langle\rangle X,\left([]^{-1}\right)_{\sqcup} X=X \sqcup[]^{-1} X,\left([]^{-1}\right)_{\sqcap} X=$ $X \sqcap[]^{-1} X$. Then for example we would have the abbreviated forms: convince: $((N \backslash S) / C P) /(\langle\rangle) \sqcup N$ and picture: $(C N / P P) /(\langle\rangle) \sqcup P P$.
} 


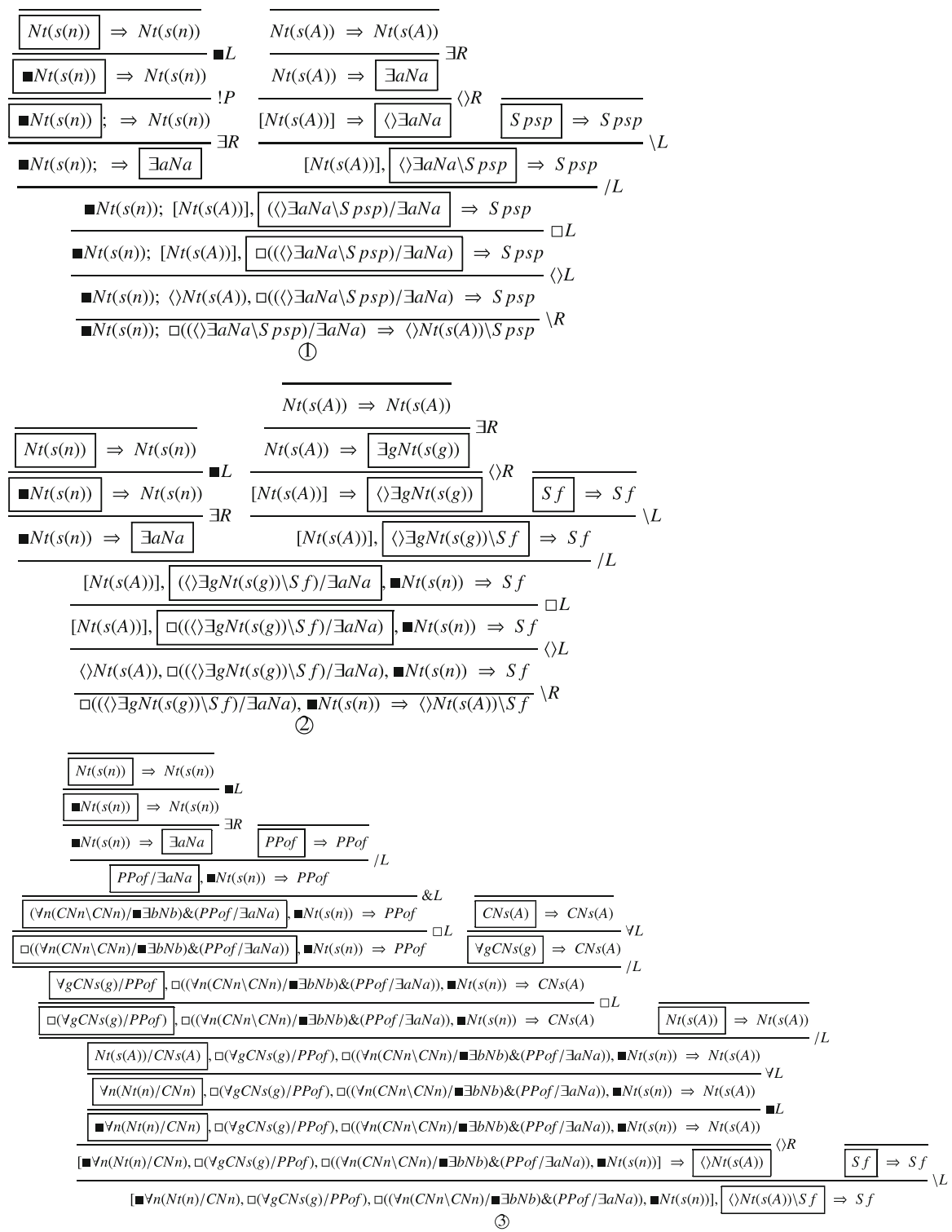

Fig. 25 Auxiliary derivations for paper that the editor of filed without reading

unbracketed; but we do not require lexical ambiguity: we can collapse the two cases into a single polymorphic indefinite type assignment: $((S \uparrow(N \sqcap\langle\rangle N)) \downarrow S) / C N{ }^{23}$

Thirdly, Levine and Hukari (2006) cite an apparent example of 'symbiotic' extraction without a host gap:

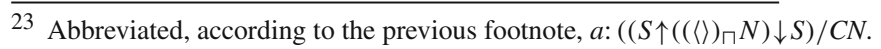




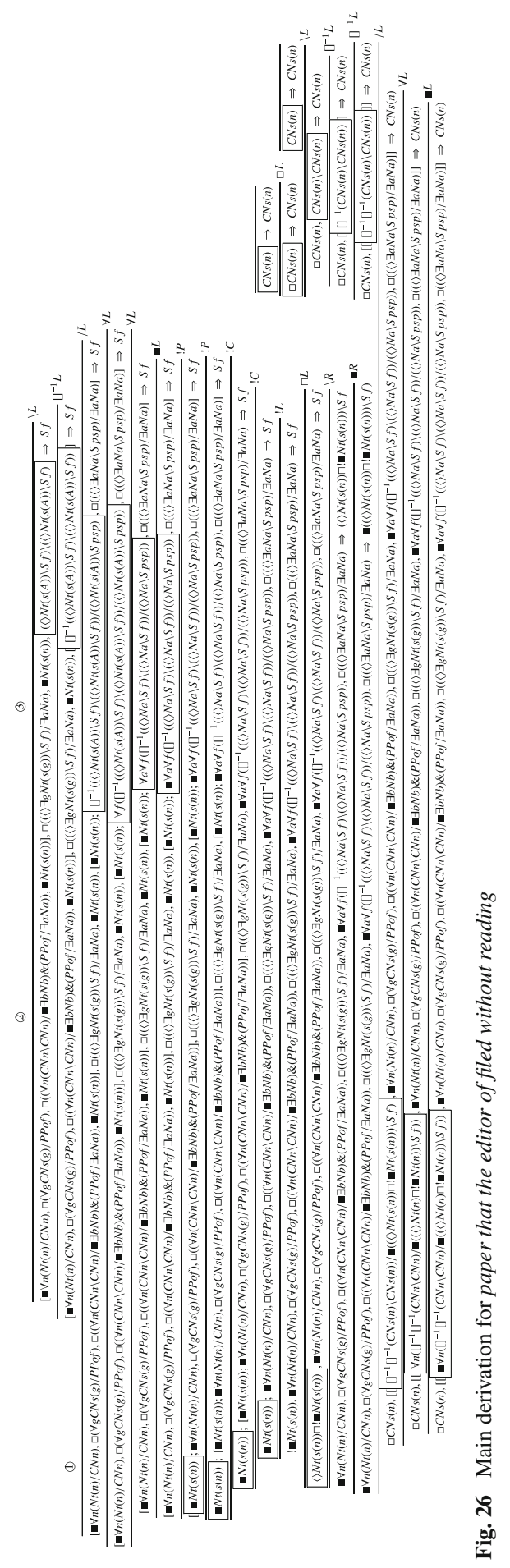


(81) people that ${ }_{i}$ fans of $t_{i}$ gather from every continent just to listen to $t_{i}$

In such a case, if the nonspecific fans has a type \langle\rangle $N / P P$ the example is generated: the object of of acts as host to the parasitic gap in the just to adverbial clause. Again we can economise type assignment to bracket inducing and non-bracket inducing fans in a single polymorphic type $(N \sqcap\langle\rangle N) / P P .^{24}$

Finally, by the same token our response to the second issue predicts the possibility of symbiotic extraction with an indefinite subject host:

(82) man that ${ }_{i}$ a friend of $t_{i}$ went to Paris without e-mailing $t_{i}$

Thus the possible exceptions to our account receive at least tentative treatment. The data regarding when (parasitic) extraction is or is not possible are complex and perhaps better accounts of the possible exceptions can be found, but we have aimed to show how at least some of this additional complexity is already within the scope of type logical grammar.

\section{Conclusion}

We have illustrated, by reference to relativisation including islands and parasitic extraction, the thesis that grammar can be reduced to logic. Our type logical categorial grammar incorporating nonassociativity, nonlinearity, and their interaction is, we suggest, mathematically interesting, technically robust, and as empirically adequate and computationally advanced as other proposals.

Acknowledgements Research partially supported by an ICREA Acadèmia 2012, SGR2014-890 (MACDA) of the Generalitat de Catalunya and MINECO project APCOM (TIN2014-57226-P). Many thanks to Stefan Kaufmann for editorial guidance, and to Linguistics and Philosophy reviewing which has greatly improved the paper, and to Oriol Valentín for insight and support. All errors are my own.

Open Access This article is distributed under the terms of the Creative Commons Attribution 4.0 International License (http://creativecommons.org/licenses/by/4.0/), which permits unrestricted use, distribution, and reproduction in any medium, provided you give appropriate credit to the original author(s) and the source, provide a link to the Creative Commons license, and indicate if changes were made.

\section{Appendix 1: Semantic representation language}

\section{Semantic types}

Recall the following operations on sets:

(83) a. Functional exponentiation: $X^{Y}=$ the set of all total functions from $Y$ to $X$

b. Cartesian product: $X \times Y=\{\langle x, y\rangle \mid x \in X \& y \in Y\}$

c. Disjoint union: $X \uplus Y=(\{1\} \times X) \cup(\{2\} \times Y)$

The set $\mathcal{T}$ of semantic types of the semantic representation language is defined on the basis of a set $\delta$ of basic semantic types as follows:

(84) $\mathcal{T}::=\delta|\top| \mathcal{T}+\mathcal{T}|\mathcal{T} \& \mathcal{T}| \mathcal{T} \rightarrow \mathcal{T}|\mathbf{M} \mathcal{T}| \mathbf{L} \mathcal{T}$

24 Orfans : $\left(\langle)_{\sqcap} N / P P\right.$ 
A semantic frame comprises a family $\left\{D_{\tau}\right\}_{\tau \in \delta}$ of nonempty basic type domains and a nonempty set $W$ of worlds. This induces a nonempty type domain $D_{\tau}$ for each type $\tau$ as follows:

$$
\begin{aligned}
D_{\top} & =\{\emptyset\} & & \text { singleton set } \\
D_{\tau_{1}+\tau_{2}} & =D_{\tau_{1}} \uplus D_{\tau_{2}} & & \text { disjoint union } \\
D_{\tau_{1} \& \tau_{2}} & =D_{\tau_{1}} \times D_{\tau_{2}} & & \text { Cartesian product } \\
D_{\tau_{1} \rightarrow \tau_{2}} & =D_{\tau_{2}}^{D_{\tau_{1}}} & & \text { functional exponentiation } \\
D_{\mathbf{M} \tau} & =W \times D_{\tau} & & \text { Cartesian product } \\
D_{\mathbf{L} \tau} & =D_{\tau}^{W} & & \text { functional exponentiation }
\end{aligned}
$$

\section{Semantic terms}

The sets $\Phi_{\tau}$ of terms of type $\tau$ for each semantic type $\tau$ are defined on the basis of sets $C_{\tau}$ of constants of type $\tau$ and denumerably infinite sets $V_{\tau}$ of variables of type $\tau$ for each type $\tau$ as follows:

$$
\begin{aligned}
& \Phi_{\tau}::=C_{\tau} \\
& \Phi_{\tau}::=V_{\tau} \\
& \Phi_{\top}::=0 \\
& \text { constants } \\
& \text { variables } \\
& \Phi_{\tau}::=\Phi_{\tau_{1}+\tau_{2}} \rightarrow V_{\tau_{1}} . \Phi_{\tau} ; V_{\tau_{2}} . \Phi_{\tau} \text { case statement } \\
& \Phi_{\tau+\tau^{\prime}}::=\iota_{1} \Phi_{\tau} \\
& \Phi_{\tau^{\prime}+\tau}::=\iota_{2} \Phi_{\tau} \\
& \Phi_{\tau}::=\pi_{1} \Phi_{\tau \& \tau^{\prime}} \\
& \Phi_{\tau}::=\pi_{2} \Phi_{\tau^{\prime} \& \tau} \\
& \Phi_{\tau \& \tau^{\prime}}::=\left(\Phi_{\tau}, \Phi_{\tau^{\prime}}\right) \\
& \Phi_{\tau}::=\left(\Phi_{\tau^{\prime} \rightarrow \tau} \Phi_{\tau^{\prime}}\right) \\
& \Phi_{\tau \rightarrow \tau^{\prime}}::=\lambda V_{\tau} \Phi_{\tau^{\prime}} \\
& \Phi_{\tau}::={ }^{\vee} \Phi_{\mathbf{L} \tau} \\
& \Phi_{\mathbf{L} \tau}::{ }^{\wedge} \Phi_{\tau} \\
& \Phi_{\tau}::={ }^{\cup} \Phi_{\mathbf{M} \tau} \\
& \Phi_{\mathbf{M} \tau}::={ }^{\cap} \Phi_{\tau} \\
& \text { dummy } \\
& \text { first injection } \\
& \text { second injection } \\
& \text { first projection } \\
& \text { second projection } \\
& \text { ordered pair formation } \\
& \text { functional application } \\
& \text { functional abstraction } \\
& \text { extensionalisation } \\
& \text { intensionalisation } \\
& \text { projection } \\
& \text { injection }
\end{aligned}
$$

Given a semantic frame, a valuation $f$ mapping each constant of type $\tau$ into an element of $D_{\tau}$, an assignment $g$ mapping each variable of type $\tau$ into an element of $D_{\tau}$, and a world $i \in W$, each term $\phi$ of type $\tau$ receives an interpretation $[\phi]^{g, i} \in D_{\tau}$ as shown in Fig. 27; the update $g[x:=d]$ is $(g-\{(x, g(x)\}) \cup\{(x, d)\}$, i.e. the function which sends $x$ to $d$ and agrees with $g$ elsewhere.

In $x . \phi, \lambda x \phi$ or ${ }^{\wedge} \phi, \phi$ is the scope of $x ., \lambda x$ or ${ }^{\wedge}$. An occurrence of a variable $x$ in a term is called free if and only if it does not fall within the scope of any $x$. or $\lambda x$; otherwise it is bound (by the closest $x$. or $\lambda x$ within the scope of which it falls). The result $\phi\left\{\psi_{1} / x_{1}, \ldots, \psi_{n} / x_{n}\right\}$ of substituting terms $\psi_{1}, \ldots, \psi_{n}$ for variables $x_{1}, \ldots, x_{n}$ of the same types respectively in a term $\phi$ is the result of simultaneously replacing by $\psi_{i}$ every free occurrence of $x_{i}$ in $\phi$. We say that $\psi$ is free for $x$ in $\phi$ if and only if no variable in $\psi$ becomes bound in $\phi\{\psi / x\}$. We say that a term is modally closed if and only if every occurrence of ${ }^{\vee}$ occurs within the scope of an ${ }^{\wedge}$. A modally closed term is denotationally invariant across worlds. We say that a term $\psi$ is modally free for $x$ in $\phi$ 
Fig. 27 Interpretation of the semantic representation language

Fig. 28 Semantic conversion laws

if and only if either $\psi$ is modally closed, or no free occurrence of $x$ in $\phi$ is within the scope of an ${ }^{\wedge}$. The laws of conversion in Fig. 28 obtain.

The so-called commuting conversions with respect to normalisation for the case statement are omitted. $\phi \rightarrow y . \psi ; z \cdot \chi=\phi \rightarrow x .(\psi\{x / y\}) ; z \cdot \chi$

if $x$ is not free in $\psi$ and is free for $y$ in $\psi$

$\phi \rightarrow y . \psi ; z \cdot \chi=\phi \rightarrow y . \psi ; x .(\chi\{x / z\})$

if $x$ is not free in $\chi$ and is free for $z$ in $\chi$

$$
\lambda y \phi=\lambda x(\phi\{x / y\})
$$

if $x$ is not free in $\phi$ and is free for $y$ in $\phi$

$\alpha$-conversion

$\iota_{1} \phi \rightarrow y \cdot \psi ; z \cdot \chi=\psi\{\phi / y\}$

if $\phi$ is free for $y$ in $\psi$ and modally free for $y$ in $\psi$ $\iota_{2} \phi \rightarrow y . \psi ; z \cdot \chi=\chi\{\phi / z\}$

if $\phi$ is free for $z$ in $\chi$ and modally free for $z$ in $\chi$

$$
\begin{aligned}
\pi_{1}(\phi, \psi) & =\phi \\
\pi_{2}(\phi, \psi) & =\psi \\
(\lambda x \phi \psi) & =\phi\{\psi / x\}
\end{aligned}
$$

$$
\begin{aligned}
& \vee \wedge \phi=\phi \\
& \cup \cap \phi=\phi
\end{aligned}
$$

$\beta$-conversion

$$
\begin{aligned}
\left(\pi_{1} \phi, \pi_{2} \phi\right) & =\phi \\
\lambda x(\phi x) & =\phi
\end{aligned}
$$

if $x$ is not free in $\phi$

$$
\wedge \vee_{\phi}=\phi
$$

if $\phi$ is modally closed

$$
\cap \cup \phi=\phi
$$

$\eta$-conversion if $\psi$ is free for $x$ in $\phi$, and modally free for $x$ in $\phi$ 


\section{Appendix 2: A lexicon}

$\mathbf{a}: \square g(\forall f((S f \uparrow \square t(s(g))) \downarrow S f) / C N s(g)): \lambda A \lambda B \exists C[(A C) \wedge(B C)]$

admire : $\square((\langle\rangle(\exists a N a-\exists g N t(s(g))) \backslash S f) / \exists a N a):{ }^{\wedge} \lambda A \lambda B$ (Pres ((`admire A) B))

And : $\square f(S f / S f): \lambda A A$

and : $\forall f\left(\left(? \square S f \backslash[]^{-1}[]^{-1} S f\right) / \boldsymbol{\square} S f\right):\left(\Phi^{n+} 0\right.$ and $)$

and : $\forall a \forall f\left(\left(? \square\left(\langle>N a \backslash S f) \backslash[]^{-1}[]^{-1}(\langle\rangle N a \backslash S f)\right) / \square(\langle\rangle N a \backslash S f)\right):\left(\Phi^{n+}\left(s_{0} 0\right)\right.\right.$ and $)$

and : $\square \forall \forall \forall f\left(\left(? \mathbb{\square}(S f / ! N a) \backslash[]^{-1}[]^{-1}(S f / ! N a)\right) / \mathbf{\square}(S f / ! N a)\right):\left(\Phi^{n+}\left(s_{0}\right)\right.$ and $)$

and : $\forall f\left(\left(? \square(S f / \exists a N a) \backslash[]^{-1}[]^{-1}(S f / \exists a N a)\right) / \square(S f / \exists a N a)\right):\left(\Phi^{n+}(s 0)\right.$ and $)$

and : $\forall w \forall a \forall b \forall f\left(\left(\square\left(\left(S f \uparrow(((\langle>N a \backslash S f) \circ W w) / N b)){ }_{2} W w\right) \backslash\right.\right.\right.$

[]$\left.^{-1}[]^{-1}\left((S f \uparrow(((\langle\rangle N a \backslash S f) \circ W w) / N b)){ }_{2} W w\right)\right) /$

- $\left.\left((S f \uparrow(((\langle\rangle a \backslash S f) \circ-W w) / N b)) \uparrow_{2} W w\right)\right): \lambda A \lambda B \lambda C[(B C) \wedge(A C)]$

and : $\forall f \forall a\left(\left(? \square((\langle\rangle N a \backslash S f) / \exists b N b) \backslash[]^{-1}[]^{-1}((\langle N a \backslash S f) / \exists b N b)) / \square((\langle\rangle N a \backslash S f) / \exists b N b)\right)\right.$ :

$\left(\Phi^{n+}(s(s 0))\right.$ and $)$

and : $\forall f \forall a\left(\left(? \square\left(((\langle>N a \backslash S f) / \exists b N b) \backslash(\langle\rangle N a \backslash S f)) \backslash[]^{-1}[]^{-1}(((\langle\rangle N \backslash S f) / \exists b N b) \backslash(\langle\rangle N a \backslash S f))\right) /\right.\right.$

$\mathbf{\square}(((\langle\rangle N a \backslash S f) / \exists b N b) \backslash(\langle>N a \backslash S f))):\left(\Phi^{n+}(s(s 0))\right.$ and $)$

and : $\forall f \forall a((\mathbf{\square}(((\langle>N a \backslash S f) /(\exists b N b \oplus \exists g((C N g / C N g) \sqcup(C N g \backslash C N g)))) \backslash(\langle\rangle N a \backslash S f)) \backslash$

[]$^{-1}[]^{-1}(((\langle N a \backslash S f) /(\exists b N b \oplus \exists g((C N g / C N g) \sqcup(C N g \backslash C N g)))) \backslash(\langle\rangle N a \backslash S f))) / \mathbf{\square}(((\langle>N a \backslash S f) /$

$(\exists b N b \oplus \exists g((C N g / C N g) \sqcup(C N g \backslash C N g)))) \backslash(\langle\rangle N a \backslash S f))): \lambda A \lambda B \lambda C \lambda D[((B C) D) \wedge((A C) D)]$

and : $\square a \forall b \forall f\left(\left(? \square\left(((\langle>N a \backslash S f) /(\exists c N c \oplus C P b)) \backslash(\langle\rangle N a \backslash S f)) \backslash[]^{-1}[]^{-1}(((\langle\rangle N a \backslash S f) /\right.\right.\right.$

$(\exists c N c \oplus C P b)) \backslash(\langle\rangle N a \backslash S f))) / \mathbf{\square}(((\langle>N a \backslash S f) /(\exists c N c \oplus C P b)) \backslash(\langle\rangle N \backslash S f)))$ :

$\left(\Phi^{n+}(s(s 0))\right.$ and $)$

and : $\forall a \forall b \forall f\left(\left(? \square\left((\langle>N a \backslash S f) / P P b) \backslash[]^{-1}[]^{-1}((\langle\rangle N a \backslash S f) / P P b)\right) / \mathbf{\square}((\langle\rangle N a \backslash S f) / P P b)\right)\right.$ :

$\left(\Phi^{n+}(s(s 0))\right.$ and $)$

and : $\forall a \forall b \forall f\left(\left(? \square(((\langle\rangle N a \backslash S f) /(\exists c N c \bullet P P b)) \backslash(\langle\rangle N a \backslash S f)) \backslash[]^{-1}[]^{-1}(((\langle\rangle N a \backslash S f) /\right.\right.$

$(\exists c N c \bullet P P b)) \backslash(\langle>N a \backslash S f))) / \mathbf{\square}(((\langle\rangle N \backslash S f) /(\exists c N c \bullet P P b)) \backslash(\langle\rangle N a \backslash S f))):$

$\left(\Phi^{n+}(s(s 0))\right.$ and $)$

and : $\forall a \forall b \forall f\left(\left(? \square((\langle\rangle N a \backslash S f) / ! N b) \backslash[]^{-1}[]^{-1}((\langle\rangle N a \backslash S f) / ! N b)\right) / \boldsymbol{\square}((\langle\rangle N a \backslash S f) / ! N b)\right)$ :

$\left(\Phi^{n+}(s(s 0))\right.$ and $)$

ate : $\square((\langle\rangle \exists a N a \backslash S f) / \exists a N a):{ }^{\wedge} \lambda A \lambda B\left(\right.$ Past $\left(\left({ }^{\top}\right.\right.$ eat $\left.\left.\left.A\right) B\right)\right)$

bagels : $\square(N t(p(n)) \& C N p(n)):{ }^{\wedge}(($ gen $`$ bagels $), `$ bagels $)$

barn : $\square C N s(n):$ barn

be : $\square((\langle\rangle W[$ there $] \multimap S b) / \exists a N a):{ }^{\wedge} \lambda A\left({ }^{(} b e A\right)$

before : $\square(\forall a \forall f((\langle\rangle N a \backslash S f) \backslash(\langle\rangle N a \backslash S f)) / S f): \lambda A \lambda B \lambda C(($ before $A)(B C))$

beginning : $\square C N s(n)$ : beginning

believes : $\square((\langle\rangle \exists g N t(s(g)) \backslash S f) /(C P t h a t \sqcup \square S f)):{ }^{\wedge} \lambda A \lambda B\left(\right.$ Pres $\left(\left({ }^{\prime}\right.\right.$ believe A) B))

bill : $N t(s(m)): b$

book : $\square C N s(n):$ book

bought : $\square\left((\langle\exists a N a \backslash S f) /(\exists a N a \bullet \exists a N a))\right.$ : $\lambda^{\lambda} A \lambda B\left(\right.$ Past $\left.\left(\left(\left(b u y \pi_{1} A\right) \pi_{2} A\right) B\right)\right)$

bought : $\square((\langle\rangle a N a \backslash S f) / \exists a N a)$ : ${ }^{\wedge} \lambda A \lambda B\left(\right.$ Past $\left(\left({ }^{\prime}\right.\right.$ buy $\left.\left.\left.A\right) B\right)\right)$

by : $\forall a(((\langle\rangle N a \backslash S-) \backslash(\langle\rangle N a \backslash S-)) / N a): \lambda A \lambda B \lambda C[[C=A] \wedge(B C)]$

by : $\square(\forall n(C N n \backslash C N n) / \exists a N a):{ }^{\wedge} \lambda A \lambda B\left(\left({ }^{\circ} b y A\right) B\right)$

buys : $\square((\langle\rangle g N t(s(g)) \backslash S f) /(\exists a N a \bullet \exists a N a)):{ }^{\wedge} \lambda A \lambda B\left(\right.$ Pres $\left.\left(\left(\left({ }^{\circ} b u y \pi_{1} A\right) \pi_{2} A\right) B\right)\right)$

calls : $\square((\langle\rangle g N t(s(g)) \backslash S f) / \exists a((W[u p] \bigcirc N a) \sqcup(N a \backslash W[u p]))):{ }^{\wedge} \lambda A \lambda B\left(\left({ }^{\circ}\right.\right.$ phone $\left.\left.A\right) B\right)$

catch : $\square((\langle\rangle \exists a N a \backslash S b) / \exists a N a):{ }^{\wedge} \lambda A \lambda B\left(\left({ }^{\prime} \operatorname{catch} A\right) B\right)$

cezanne : $N t(s(m)): c$ 


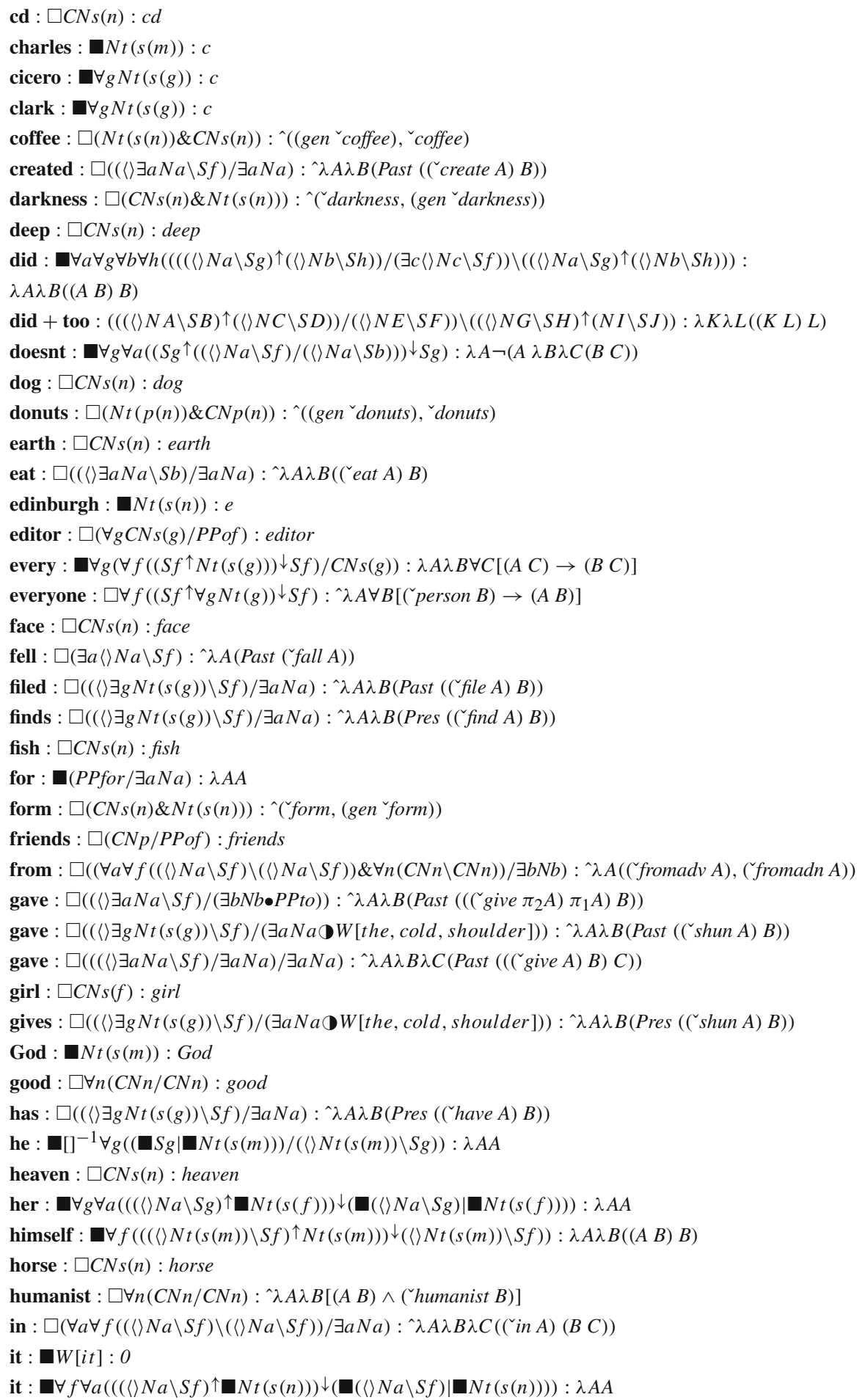




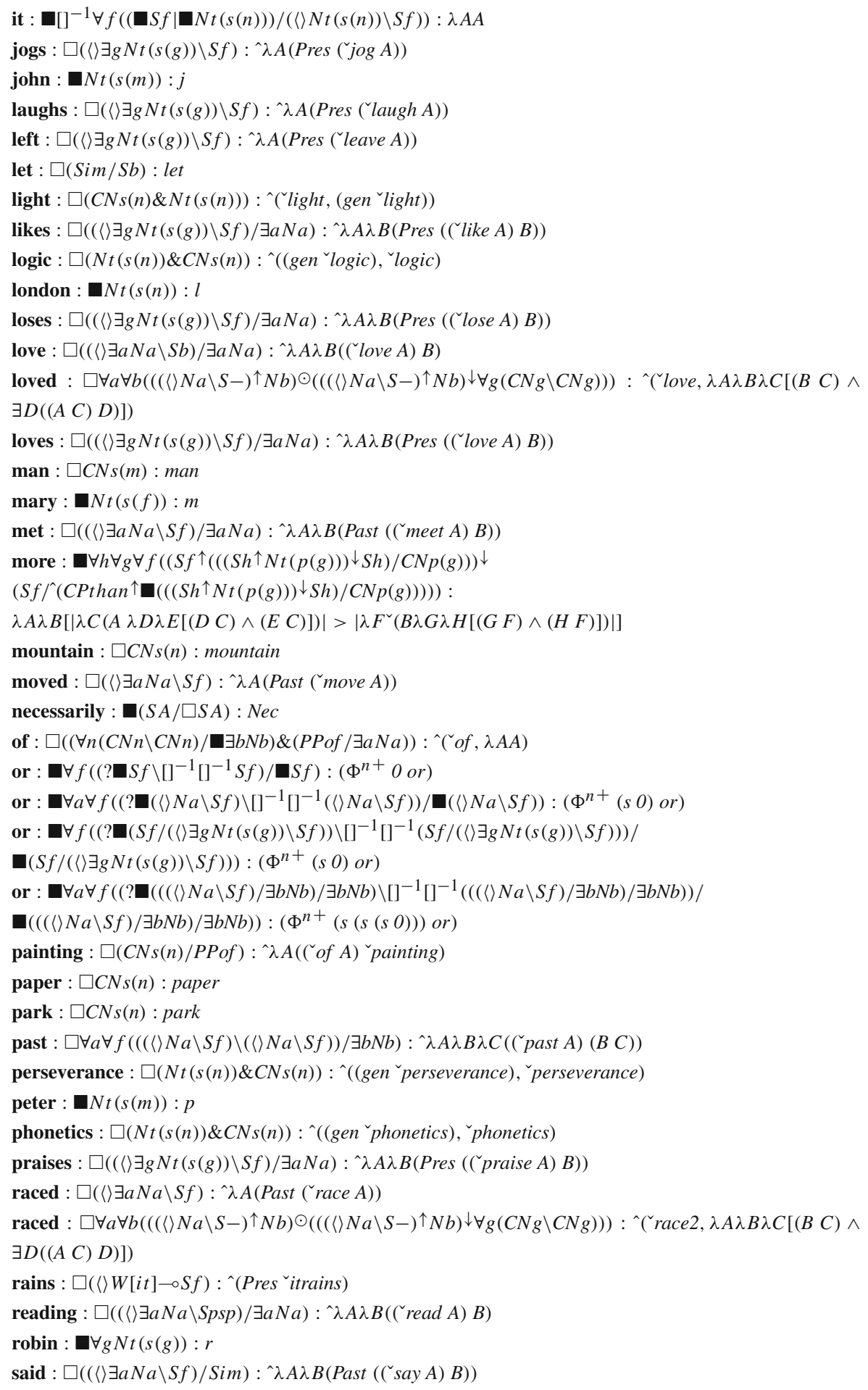


saw : $\square((\langle\rangle \exists a N a \backslash S f) /(\exists a N a \oplus C P$ that $)):{ }^{\wedge} \lambda A \lambda B($ Past $((A \rightarrow C$. ( ( seee C $) ;$ D.( ( seet D) $\left.) B)\right)$

seeks : $\square((\langle\rangle \exists g N t(s(g)) \backslash S f) / \square \forall a \forall f(((N a \backslash S f) / \exists b N b) \backslash(N a \backslash S f))):{ }^{\wedge} \lambda A \lambda B\left(\left({ }^{\sim}\right.\right.$ tries $^{\wedge}\left(\left({ }^{\wedge} A^{\vee}\right.\right.$ find $\left.\left.\left.) B\right)\right) B\right)$ sees : $\square((\langle\rangle \exists g N t(s(g)) \backslash S f) / \exists a N a):{ }^{\wedge} \lambda A \lambda B($ Pres $((\ulcorner$ see A) B))

sent : $\square((\langle\rangle \exists a N a \backslash S f) /(\exists b N b \bullet P P t o)):{ }^{\wedge} \lambda A \lambda B\left(\right.$ Past $\left(\left(\left(\right.\right.\right.$ sent $\left.\left.\left.\left.\pi_{2} A\right) \pi_{1} A\right) B\right)\right)$

sent : $\square\left(((\langle\exists \exists a N a \backslash S f) / \exists a N a) / \exists a N a):{ }^{\wedge} \lambda A \lambda B \lambda C(\right.$ Past $(((`$ send $A) B) C))$

she : $\boldsymbol{\square}^{-1} \forall g((\boldsymbol{\square} S g \mid \boldsymbol{\square} N t(s(f))) /(\langle\rangle N t(s(f)) \backslash S g)): \lambda A A$

sings : $\square(\langle\rangle \exists g N t(s(g)) \backslash S f)$ : ${ }^{\wedge} \lambda A($ Pres $(\ulcorner$ sing $A))$

slept : $\square(\langle\rangle \exists g N t(s(g)) \backslash S f):{ }^{\wedge} \lambda A($ Past (`sleep $\left.A)\right)$

slowly : $\square \forall a \forall f(\square(\langle\rangle N a \backslash S f) \backslash(\langle\rangle \square N a \backslash S f))$ : ^ $\lambda A \lambda B\left({ }^{\wedge}\right.$ slowly^(`A`B))

sneezed : $\square(\langle\rangle \exists g N t(s(g)) \backslash S f):{ }^{\wedge} \lambda A$ (Past (`sneeze A))

sold : $\square((\langle\rangle \exists a N a \backslash S f) /(\exists b N b \bullet P P f o r))$ : ${ }^{\wedge} \lambda A \lambda B\left(\right.$ Past $\left(\left(\left({ }^{\prime}\right.\right.\right.$ sell $\left.\left.\left.\left.\pi_{2} A\right) \pi_{1} A\right) B\right)\right)$

someone : $\square \forall f((S f \uparrow \square \forall g N t(g)) \downarrow S f):{ }^{\wedge} \lambda A \exists B\left[\left({ }^{\wedge}\right.\right.$ person $\left.\left.B\right) \wedge(A B)\right]$

Spirit : $\square C N s(m)$ : Spirit

studies : $\square((\langle\rangle \exists g N t(s(g)) \backslash S f) / \exists a N a)$ : ^ $\lambda A \lambda B($ Pres ((`study A) B))

such+that : $\forall n((C N n \backslash C N n) /(S f \mid \square N(n))): \lambda A \lambda B \lambda C[(B C) \wedge(A C)]$

suzy : $N t(s(f)): s$

talks : $\square(\langle\rangle \exists g N t(s(g)) \backslash S f):{ }^{\wedge} \lambda A\left(\right.$ Pres $\left({ }^{2}\right.$ talk $\left.\left.A\right)\right)$

tall : $\square \forall g(C N g / C N g):$ tall

tenmilliondollars : $\square N t(s(n))$ : tenmilliondollars

than : $\square($ CPthan $/ \square S f): \lambda A A$

that : $\square($ CPthat $/ \square S f): \lambda A A$

that : $\square \forall n\left([]^{-1}[]^{-1}(C N n \backslash C N n) / \mathbf{\square}((\langle\rangle t(n) \sqcap ! \square N t(n)) \backslash S f)\right): \lambda A \lambda B \lambda C[(B C) \wedge(A C)]$

the : $\forall n(N t(n) / C N n): \iota$

the+cold+shoulder : $\mathbf{\square} W[$ the, cold, shoulder $]: 0$

there : $\square W[$ there $]: 0$

thinks : $\square((\langle\rangle \exists g N t(s(g)) \backslash S f) /(C P$ that $\sqcup \square S f))$ : ${ }^{\wedge} \lambda A \lambda B\left(\right.$ Pres $\left(\left({ }^{\circ}\right.\right.$ think $\left.\left.\left.A\right) B\right)\right)$

to : $\mathbf{\square}((P P t o / \exists a N a) \sqcap \forall n((\langle\rangle N n \backslash S i) /(\langle\rangle N n \backslash S b))): \lambda A A$

today : $\square \forall a \forall f((\langle\rangle N a \backslash S f) \backslash(\langle\rangle N a \backslash S f)):{ }^{\wedge} \lambda A \lambda B\left({ }^{\wedge}\right.$ today $\left.(A B)\right)$

tries : $\square((\langle\rangle \exists g N t(s(g)) \backslash S f) / \square(\langle\rangle \exists g N t(s(g)) \backslash S i)):{ }^{\wedge} \lambda A \lambda B\left(\left({ }^{\wedge}\right.\right.$ tries $\left.\left.{ }^{\wedge}\left({ }^{\wedge} A B\right)\right) B\right)$

tully : $\mathbf{\square} t(s(m)): t$

unicorn : $\square C N s(n)$ : unicorn

up : $\mathbf{\square} W[u p]: 0$

upon : $\square((\forall b \forall f((\langle\rangle N b \backslash S f) \backslash(\langle\rangle N b \backslash S f)) \& \forall g(C N g \backslash C N g)) / \exists a N a)$ : ^ $\lambda A(($ uponadv $A),(($ uponadn $A))$

void : $\square \forall g(C N g / C N g)$ : void

walk : $\square(\langle\rangle(\exists a N a-\exists g N t(s(g))) \backslash S f):{ }^{\wedge} \lambda A($ Pres $(\ulcorner$ walk $A))$

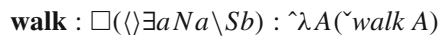

walks : $\square(\langle\rangle \exists g N t(s(g)) \backslash S f)$ : $\lambda^{\lambda} A($ Pres (`walk $\left.A)\right)$

was : $\square((\langle\rangle \exists g N t(s(g)) \backslash S f) /(\exists a N a \oplus(\exists g((C N g / C N g) \sqcup(C N g \backslash C N g))-I)))$ :

$\lambda A \lambda B($ Past $(A \rightarrow C$. $[B=C] ; D .((D \lambda E[E=B]) B)))$

was : $\square((\langle\rangle W[$ there $] \multimap S f) / \exists a N a):{ }^{\wedge} \lambda A($ Past $(\ulcorner$ be A))

waters : $\square C N p(n):$ waters

which : $\square \forall n \forall m\left(\left(N t(n)^{\uparrow} N t(m)\right)^{\downarrow}\left([]^{-1}[]^{-1}(C N m \backslash C N m) / \square((\langle\rangle N t(n) \sqcap ! \square N t(n)) \backslash S f)\right)\right)$ :

$\lambda A \lambda B \lambda C \lambda D[(C D) \wedge(B(A D))]$

who : $\square \forall \forall \forall n\left([]^{-1}[]^{-1}(N t(n) \backslash((S h \uparrow N t(n)) \downarrow S h)) / \square((\langle\rangle N t(n) \sqcap ! \square N t(n)) \backslash S f)\right)$ :

$\lambda A \lambda B \lambda C[(A B) \wedge(C B)]$

will : $\square a((\langle\rangle N a \backslash S f) /(\langle\rangle N a \backslash S b)): \lambda A \lambda B(F u t(A B))$ 


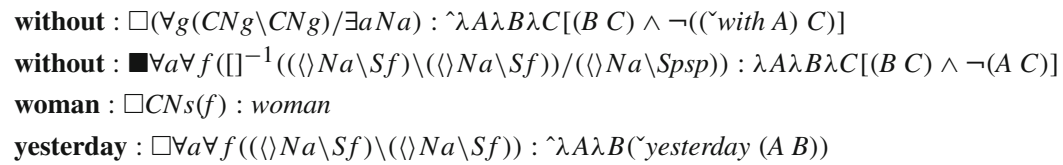

\section{References}

Ades, A. E., \& Steedman, M. J. (1982). On the order of words. Linguistics and Philosophy, 4, 517-558. Ajdukiewicz, K. (1935). Die syntaktische Konnexität. Studia Philosphica,1, 1-27, 1935. (Translated in Polish logic: 1920-1939, pp. 207-231, by S. McCall, Ed., 1967, Oxford: Oxford University Press.)

Andreoli, J. M. (1992). Logic programming with focusing in linear logic. Journal of Logic and Computation, 2(3), 297-347.

Barker, C., \& Shan, C. (2015). Continuations and natural language. New York: Oxford University Press.

Barry, G., Hepple, M., Leslie, N., \& Morrill, G. (1991). Proof figures and structural operators for categorial grammar. In Proceedings of the fifth conference of the European Chapter of the Association for Computational Linguistics (pp. 198-203). Berlin.

Carpenter, B. (1997). Type-logical semantics. Cambridge, MA: MIT.

Chomsky, N. (1973). Conditions on transformations. In S. Anderson \& P. Kiparsky (Eds.), A Festschrift for Morris Halle (pp. 232-286). New York: Holt, Rinehart and Winston.

Deane, P. (1991). Limits to attention: A cognitive theory of island phenomena. Cognitive Linguistics, 2(1), $1-63$.

Engdahl, E. (1983). Parasitic gaps. Linguistics and Philosophy, 6, 5-34.

Gentzen, G. (1934). Untersuchungen über das logische Schliessen. Mathematische Zeitschrift, 39, 176-210 and 405-431. (Translated in The collected papers of Gerhard Gentzen, pp. 68-131, by M. E. Szabo, Ed., 1969, Amsterdam: North-Holland.)

Girard, J.-Y. (1987). Linear logic. Theoretical Computer Science, 50, 1-102.

Girard, J.-Y. (2011). The blind spot. Zürich: European Mathematical Society.

Hepple, M. (1990). The grammar and processing of order and dependency. $\mathrm{Ph} . \mathrm{D}$. Thesis, University of Edinburgh.

Hofmeister, H., Casasanto, L. S., \& Sag, I. (2013). Islands in the grammar? Standards of evidence. In D. Forget, P. Hirchbuhler, F. Martineau, \& M.-L. Rivero (Eds.), Experimental syntax and island effects (pp. 42-63). Cambridge: CUP.

Hofmeister, P., \& Sag, I. A. (2010). Cognitive constraints and island effects. Language, 86(2), 366-415.

Jäger, G. (2005). Anaphora and type logical grammar (Vol. 24). Trends in logic-Studia Logica Library. Dordrecht: Springer.

Jansche, M., \& Vasishth, S. (2002). Review of Mark Steedman, the syntactic process. Journal of Linguistics, $38,684-690$.

Kanazawa, M. (1992). The Lambek calculus enriched with additional connectives. Journal of Logic, Language and Information, 1, 141-171.

Kanovich, M., Kuznetsov, S., \& Scedrov, A. (2016). Undecidability of the Lambek calculus with a relevant modality. In A. Foret, G. Morrill, R. Muskens, R. Osswald, \& S. Pogodalla (Eds.), Formal grammar 2015: Revised selected papers. Formal grammar 2016: Proceedings. LNCS (Vol. 9804, pp. 240-256) Berlin. Springer.

Kehler, A. (2002). Coherence, reference and the theory of grammar. Stanford, CA: CSLI.

Kluender, R. (1992). Deriving island constraints from principles of predication. In H. Goodluck \& M. Rochemont (Eds.), Island constraints: Theory, acquisition, and processing (pp. 223-258). Dordrecht: Kluwer.

Kluender, R. (1998). On the distinction between strong and weak islands: A processing perspective. In P. Culicover \& L. McNally (Eds.), The limits of syntax. Syntax and semantics (Vol. 29, pp. 241-279). San Diego: Academic Press.

Kubota, Y., \& Levine, R. (2015). Against ellipsis: Arguments for the direct licensing of 'non-canonical' coordinations. Linguistics and Philosophy, 38(6), 521-576. 
Lakoff, G. (1986). Frame semantic control of the coordinate structure constraint. In A. M. Farley, P. T. Farley, \& K.-E. McCullough (Eds.), CLS 22 PART 2: Papers from the parasession on pragmatics and grammatical theory (Vol. 152). Chicago: Chicago Linguistics Society.

Lambek, J. (1958). The mathematics of sentence structure. American Mathematical Monthly, 65, 154-170.

Lambek, J. (1961). On the calculus of syntactic types. In R. Jakobson (Ed.), Structure of language and its mathematical aspects, Proceedings of the symposia in applied mathematics XII (pp. 166-178). Providence, RI: American Mathematical Society.

Lambek, J. (1988). Categorial and categorical grammars. In R. T. Oehrle, E. Bach, \& D. Wheeler (Eds.), Categorial grammars and natural language structures. Studies in linguistics and philosophy (Vol. 32, pp. 297-317). Dordrecht: D. Reidel.

Levine, R. D., \& Hukari, T. E. (2006). The unity of unbounded dependency constructions. Stanford, CA: CSLI.

Mihaliček, V., \& Pollard, C. (2012). Distinguishing phenogrammar from tectogrammar simplifies the analysis of interrogatives. In P. de Groote \& M.-J. Nederhof (Eds.), Formal grammar 2010/2011 (pp. 130-145). Heidelberg: Springer.

Moortgat, M. (1988). Categorial investigations: Logical and linguistic aspects of the Lambek Calculus, Foris, Dordrecht. Ph.D. Thesis, Universiteit van Amsterdam.

Moortgat, M. (1995). Multimodal linguistic inference. Journal of Logic, Language and Information, 5(3,4), 349-385, 1996. (Also in Bulletin of the IGPL,3(2,3), 371-401, 1995.)

Moortgat, M. (1997). Categorial type logics. In J. van Benthem \& A. ter Meulen (Eds.), Handbook of logic and language (pp. 93-177). Amsterdam/Cambridge, MA: Elsevier/MIT.

Moot, R., \& Retoré, C. (2012). The logic of categorial grammars: A deductive account of natural language syntax and semantics. Heidelberg: Springer.

Morrill, G. (1990a). Grammar and logical types. In M. Stockhof \& L. Torenvliet (Eds.), Proceedings of the seventh Amsterdam colloquium (pp. 429-450). Amsterdam: ILLC.

Morrill, G. (1990b). Intensionality and boundedness. Linguistics and Philosophy, 13(6), 699-726.

Morrill, G. (1992). Categorial formalisation of relativisation: Pied piping, islands, and extraction sites. Technical Report LSI-92-23-R. Departament de Llenguatges i Sistemes Informàtics, Universitat Politècnica de Catalunya.

Morrill, G. (1994). Type logical grammar: Categorial logic of signs. Dordrecht: Kluwer.

Morrill, G. (2000). Incremental processing and acceptability. Computational Linguistics, 26(3), 319-338.

Morrill, G. (2011a). Logic programming of the displacement calculus. In S. Pogodalla \& J.-P. Prost (Eds.), Proceedings of logical aspects of computational linguistics 2011, LACL'11, Montpellier. LNAI 6736 in Springer lecture notes in AI (pp. 175-189). Berlin: Springer.

Morrill, G. (2011b). Categorial grammar: Logical syntax, semantics, and processing. New York: Oxford University Press.

Morrill, G. (2012). Logical grammar. In R. Kempson, T. Fernando, \& N. Asher (Eds.), The handbook of philosophy of linguistics (pp. 63-92). Oxford: Elsevier.

Morrill, G., \& Merenciano, J.-M. (1996). Generalising discontinuity. Traitement automatique des langues, 37(2), 119-143.

Morrill, G., \& Valentín, O. (2010). Displacement calculus. Linguistic Analysis, 36(1-4), 167-192. Special issue Festschrift for Joachim Lambek. arXiv.1004.4181.

Morrill, G., \& Valentín, O. (2014a). Displacement logic for anaphora. Journal of Computing and System Science, 80, 390-409. doi:10.1016/j.jcss.2013.05.006.

Morrill, G., \& Valentín, O. (2014b). Semantically inactive multiplicatives and words as types. In N. Asher \& S. Soloviev (Eds.), Proceedings of logical aspects of computational linguistics, LACL'14, Toulouse. LNCS, FoLLI Publications on logic, language and information (Vol. 8535, pp. 149-162). Berlin: Springer.

Morrill, G., \& Valentín, O. (2015a). Computational coverage of TLG: Nonlinearity. In M. Kanazawa, L. S. Moss, \& V. de Paiva (Eds.), Proceedings of NLCS'15. Third workshop on natural language and computer science. EPiC (Vol. 32, pp. 51-63), Kyoto, 2015. Workshop affiliated with Automata, Languages and Programming (ICALP) and Logic in Computer Science (LICS).

Morrill, G., \& Valentín, O. (2015b). Multiplicative-additive focusing for parsing as deduction. In I. Cervesato \& C. Schürmann (Eds.), First international workshop on focusing, workshop affiliated with LPAR 2015. EPTCS (Vol. 197, pp. 29-54). Fiji: Suva.

Morrill, G., \& Valentín, O. (2016). On the logic of expansion in natural language. In M. Amblard, P. de Groote, S. Pogodalla, \& C. Retoré (Eds.), Proceedings of logical aspects of computational linguistics, 
LACL'16, Nancy. LNCS, FoLLI Publications on logic, language and information (Vol. 10054, pp. 228-246). Berlin: Springer.

Morrill, G., Valentín, O., \& Fadda, M. (2009). Dutch grammar and processing: A case study in TLG. In P. Bosch, D. Gabelaia, \& J. Lang (Eds.), Logic, language, and computation: 7th international Tbilisi symposium, revised selected papers. Lecture notes in artificial intelligence (Vol. 5422, pp. 272-286). Berlin: Springer.

Morrill, G., Valentín, O., \& Fadda, M. (2011). The displacement calculus. Journal of Logic, Language and Information, 20(1), 1-48. doi:10.1007/s10849-010-9129-2.

Muskens, R. (2003). Language, lambdas and logic. In G.-J. M. Kruijff \& R. T. Oehrle (Eds.), Resourcesensitivity in binding and anaphora. Studies in linguistics and philosophy (Vol. 80, pp. 23-54). Dordrecht: Kluwer.

Newmeyer, F. J. (2016). Nonsyntactic explanations of island constraints. Annual Review of Linguistics, 2, 187-210.

Postal, P. M. (1993). Parasitic gaps and across-the-board phenomenon. Linguistic Inquiry, 24(4), 735-754.

Sag, I. A. (1983). On parasitic gaps. Linguistics and Philosophy, 6, 35-45.

Sprouse, J., Wagers, M., \& Phillips, C. (2012). A test of the relation between working memory capacity and syntactic island effects. Language, $88,82-123$.

Steedman, M. (1987). Combinatory grammars and parasitic gaps. Natural Language and Linguistic Theory, 5, 403-439.

Szabolcsi, A. (1983). ECP in categorial grammar. Manuscript. Nijmegen: Max Planck Institute.

Szabolcsi, A. (2006). Strong versus weak islands. In M. Everaert \& H. van Riemsdijk (Eds.), The Blackwell companion to syntax (pp. 479-531). Oxford: Blackwell.

Taraldsen, T. (1979). The theoretical interpretation of a class of marked extractions. In A. Belleti, L. Brandi, \& L. Rizzi (Eds.), Theory of markedness in generative grammar. Scuole Normal Superiore de Pisa: Pisa.

Valentín, O. (2012). Theory of discontinuous Lambek calculus. Ph.D. Thesis, Universitat Autònoma de Barcelona, Barcelona

Valentín, O. (2014). The hidden structural rules of the discontinuous Lambek calculus. In C. Casadio, B. Coeke, M. Moortgat, \& P. Scott (Eds.), Categories and types in logic, language and physics: Essays dedicated to Jim Lambek on the occasion of his 90th birthday. LNCS, FoLLI Publications in logic, language and information (Vol. 8222, pp. 402-420). Berlin: Springer.

Valentín, O., Serret, D., \& Morrill, G. (2013). A count invariant for Lambek calculus with additives and bracket modalities. In G. Morrill \& M.-J. Nederhof (Eds.), Proceedings of formal grammar 2012 and 2013. LNCS, FoLLI Publications in logic, language and information (Vol. 8036, pp. 263-276). Berlin: Springer.

van Benthem, J. (1991). Language in action: Categories, lambdas, and dynamic logic. Studies in logic and the foundations of mathematics (Vol. 130). Amsterdam. Revised student edition printed in 1995 by the MIT. 